\title{
Interplay between low-lying isoscalar and isovector dipole modes: A comparative analysis between semiclassical and quantum approaches
}

\author{
S. Burrello, ${ }^{1}$ M. Colonna, ${ }^{1}$ G. Colò, ${ }^{2,3}$ D. Lacroix, ${ }^{4}$ X. Roca-Maza,,${ }^{2,3}$ G. Scamps,${ }^{5,6}$ and H. Zheng ${ }^{1,7}$ \\ ${ }^{1}$ Laboratori Nazionali del Sud, INFN, I-95123 Catania, Italy \\ ${ }^{2}$ Dipartimento di Fisica, Università degli Studi di Milano, via Celoria 16, Milano 20133, Italy \\ ${ }^{3}$ Istituto Nazionale di Fisica Nucleare (INFN), Sezione di Milano, via Celoria 16, Milano 20133, Italy \\ ${ }^{4}$ Institut de Physique Nucléaire, IN2P3-CNRS, Université Paris-Sud, Université Paris-Saclay, F-91406 Orsay Cedex, France \\ ${ }^{5}$ Institute of Astronomy and Astrophysics (IAA), Université libre de Bruxelles (ULB), CP 226, \\ Boulevard du Triomphe, B-1050 Bruxelles, Belgium \\ ${ }^{6}$ Center for Computational Sciences, University of Tsukuba, Tsukuba 305-8571, Japan \\ ${ }^{7}$ School of Physics and Information Technology, Shaanxi Normal University, Xi'an 710119, China
}

(Received 22 February 2019; published 13 May 2019)

\begin{abstract}
We perform time-dependent Hartree-Fock (TDHF) calculations, employing Skyrme functionals, to investigate the small amplitude dipole response of selected neutron-rich nuclei and $\mathrm{Sn}$ isotopes. A detailed comparison with the dipole strength predicted by random-phase approximation calculations is presented for the first time. TDHF results are also confronted to Vlasov calculations, to explore up to which extent a semiclassical picture can explain the properties of the nuclear response. The focus is on the low-energy response, below the giant dipole resonance region, where different modes of nonnegligible strength are identified. We show that the relative weight of these excitations evolves with nuclear global features, such as density profile and neutron skin, which in turn reflect important properties of the nuclear effective interaction. A thorough analysis of the associated transition densities turns out to be quite useful to better characterize the mixed isoscalar (IS)-isovector (IV) nature of the different modes and their surface/volume components. In particular, we show that the dipole response in the so-called pygmy dipole resonance region corresponds to isoscalarlike surface oscillations, of larger strength in nuclei with a more diffuse surface. The ratio between the IV and IS energy-weighted sum rule fractions exhausted in this region is shown to almost linearly increase with the neutron skin thickness in Sn isotopes.
\end{abstract}

DOI: 10.1103/PhysRevC.99.054314

\section{INTRODUCTION}

The development of collective motion, i.e., a coherent pattern of particles in phase-space, is a fundamental feature of many-body systems. For instance, atomic nuclei are strongly correlated, self-bound many-body systems, which, together with single-particle properties, exhibit a variety of collective phenomena [1,2]. More recent examples are provided by Bose-Einstein condensates [3-5] and there are strong experimental and theoretical evidences that similar effects occur in other fermionic systems as well [6,7]. Collective patterns may emerge out of single-particle motion whenever favored by energy and kinematic conditions. The collective dynamics is often well described at the classical level and it is reflected in the spectral properties of the corresponding quantum manybody system. Hence, the associated spectrum of excitations usually includes states of single-particle and of collective natures, together with mixed forms, with a partial degree of collectivity. A thorough understanding of the emergence of collective motion from the microscopic point of view is, however, a true challenge.

The isovector giant dipole resonance (GDR) in heavy nuclei is a prominent and well-known example of collective motion, first measured in photoabsorption experiments [8]. The cross-section associated with the electric dipole radiation and the corresponding strength function show between 10 and $30 \mathrm{MeV}$ - the energy depending on the size of the nucleus as $\approx \mathrm{A}^{1 / 3}$ - a large increase, with a spreading width larger than the mean level spacing. This excitation can be described in terms of the classical picture of neutrons and protons moving against each other, resulting in a large response function. Thus, the difference between the center-of-mass coordinates of the two spheres appears as the proper collective coordinate in this case.

In recent years, there has been a considerable amount of experimental and theoretical studies on dipole excitations in neutron-rich nuclei, and in particular on the low-energy tail of the isovector GDR, the so-called pygmy dipole strength (PDS) observed in the IV dipole response [often denominated pygmy dipole resonance (PDR)] [9-11].

The PDR has been often interpreted as an exotic mode of excitation due to the motion of the weakly bound neutron excess against an almost inert proton-neutron core [12-14], although this picture, and the underlying collective nature of the mode, are still under debate [15].

One major reason for the recently increased interest in the PDR is the possibility of carrying out several measurements on these low-lying dipole excitations, using heavy-ion $[16,17]$, proton $[18,19]$, and $\alpha$ inelastic scattering experiments $[20,21]$. Indeed, the experimental study of the PDR with 
different probes provides intimate information about the isospin nature of these excitations which would not be possible to infer from $\gamma$ experiments alone [12]. These experimental discoveries were followed by intensive theoretical investigations, focusing on the isoscalar (IS)-isovector (IV) character of dipole excitations in isospin-asymmetric nuclear systems [22,23].

In this paper we aim at getting a deeper insight into the features of the dipole response in nuclei, with a special attention to the role of neutron/proton imbalance. By looking at the dynamical response of the system to different kinds of external perturbations, we explore spatial profile and IS-IV character of the dipole excitation modes in neutron-rich nuclei and $\mathrm{Sn}$ isotopes.

This study is tackled by investigating the small amplitude limit of the dynamical nuclear response to a dipole operator within the quantal time-dependent Hartree-Fock (TDHF) method [24-26], its zero amplitude limit, known as the random-phase approximation (RPA) [27], as well as within its semiclassical analog: the Vlasov equation [28-30].

As a key point, the paper presents, for the first time, a detailed comparison between TDHF and RPA calculations, with the purpose of verifying numerically the analytical equivalence of the two approaches in the small amplitude limit. In such a way, we aim at bringing out the possible emergence of spurious differences arising from technical details and assessing practical advantages or drawbacks of the two procedures. It is worth noting that a similar task, namely carrying out detailed comparisons between the results of time-dependent relativistic mean-field, in the small-amplitude limit, and relativistic RPA, was performed in Refs. [31-33].

Examining analogies and differences between semiclassical and quantal results, one expects to learn more about the nature and the degree of collectivity of excitation modes of present experimental interest. A schematic interpretation of nuclear excitations in terms of collective motion, whenever possible, may allow one to establish a more direct connection to global features of the nuclear effective interaction, such as surface tension and symmetry energy, also linking the nuclear response to macroscopic properties of nuclei, like density profile and neutron skin [34]. To this purpose, we will also examine the sensitivity of the dipole response to specific ingredients of the nuclear mean-field potential and to the equation of state (EoS), adopting Skyrme parametrizations which mainly differ in the isovector channel [35], already employed in recent structure studies [36].

Hence, from our analysis, we also aim at extracting important information on some aspects of effective interaction and nuclear EoS of considerable relevance also in other fields, such as heavy-ion reactions and nuclear astrophysics. Last, we note that, whereas we concentrate on dipole excitations in the present work, the same investigation can be extended to other multipolarities as well (cf., e.g., Ref. [37]).

The paper is organized as follows: in Sec. II we introduce the approaches employed in our analysis and the details of the calculations related to the dipole response. Section III is devoted to the discussion of the results obtained for dipole strengths and transition densities of selected neutron-rich nuclei and Sn isotopes. We discuss in particular the features of the low-lying region of the dipole response, showing interesting connections between isoscalar and isovector strengths in neutron-rich nuclei. The paper ends up with a summary and some perspectives in Sec. IV.

\section{THEORETICAL FRAMEWORK}

\section{A. Microscopic approaches and effective interactions}

In the present work, we compare three different microscopic theories that are widely used to describe many-body dynamics: the TDHF, the RPA, and the Vlasov approaches. Main features and connections among them are briefly discussed below.

In the TDHF theory, the evolution of the one-body density matrix $\rho(t)$ is determined by

$$
i \hbar \partial_{t} \rho(t)=[h[\rho], \rho(t)],
$$

where $h[\rho]=\mathbf{p}^{2} / 2 m+U[\rho]$ is the nonrelativistic singleparticle Hamiltonian with $U[\rho]$ being the self-consistent mean-field potential, that is a functional of the one-body density.

The TDHF approach is nowadays widely used in nuclear physics to describe various aspects of nuclear dynamics [24-26,38]. Here we will restrict ourselves to the study of small deviations from the equilibrium density $\rho_{e}$. The small amplitude fluctuations $\delta \rho(t)=\rho(t)-\rho_{e}$ can be determined either by solving explicitly the time-dependent evolution given by Eq. (1) or by linearizing the TDHF equation, leading to the RPA approach. Keeping only terms linear in $\delta \rho$, Eq. (1) equals to

$$
i \hbar \frac{\partial}{\partial t} \delta \rho=\left[h\left[\rho_{e}\right], \delta \rho\right]+\left[\frac{\partial U}{\partial \rho} \cdot \delta \rho, \rho_{e}\right],
$$

so one can access the response function of the system to a small external perturbation. Therefore, despite the TDHF has a larger domain of applicability, from the analytical point of view, it is equivalent in the small amplitude regime to the RPA approach, where the time-evolution is replaced by an eigenvalue problem. A detailed discussion on the RPA method employed to obtain the results presented here can be found in Ref. [39] and references therein.

The Vlasov equation, which describes the time evolution of the one-body distribution function in phase space, represents instead the semiclassical limit of TDHF and, for smallamplitude dynamics, of the RPA equations [27]. This selfconsistent approach is suitable to describe robust quantum modes, of zero-sound type, in both nuclear matter and finite nuclei [29,40-42], though it is unable to account for effects associated with the shell structure. Expliciting the two species constituting nuclear matter, one has essentially to solve the two coupled Vlasov kinetic equations for the neutron and proton distribution functions $f_{q}(\mathbf{r}, \mathbf{p}, t)$, with $q=n, p$ [40]:

$$
\frac{\partial f_{q}}{\partial t}+\frac{\partial \epsilon_{q}}{\partial \mathbf{p}} \frac{\partial f_{q}}{\partial \mathbf{r}}-\frac{\partial \epsilon_{q}}{\partial \mathbf{r}} \frac{\partial f_{q}}{\partial \mathbf{p}}=0 .
$$

In the equations above, $\epsilon_{q}$ represents the neutron or proton single particle energy, which contains the mean-field potential $U_{q}$. 
To represent the nuclear effective interaction, we start considering a given energy density functional $\mathscr{E}[\rho]$. This formulation is very convenient since it allows to extract the mean-field potential as its functional derivative with respect to the density. Actually, the residual interaction, i.e., the antisymmetrized particle-hole interaction used in RPA calculations, is calculated via the second functional derivative of $\mathscr{E}[\rho]$, when dealing with density dependent forces.

Considering a standard Skyrme interaction, and specializing to even-even systems, the functional $\mathscr{E}[\rho]$ is expressed in terms of the isoscalar, $\rho=\rho_{n}+\rho_{p}$, and isovector, $\rho_{3}=\rho_{n}-$ $\rho_{p}$, densities and kinetic energy densities $\left(\tau=\tau_{n}+\tau_{p}, \tau_{3}=\right.$ $\left.\tau_{n}-\tau_{p}\right)$ as [43]:

$$
\begin{aligned}
\mathscr{E}= & \frac{\hbar^{2}}{2 m} \tau+C_{0} \rho^{2}+D_{0} \rho_{3}^{2}+C_{3} \rho^{\alpha+2}+D_{3} \rho^{\alpha} \rho_{3}^{2}+C_{\text {eff }} \rho \tau \\
& +D_{\text {eff }} \rho_{3} \tau_{3}+C_{\text {surf }}(\nabla \rho)^{2}+D_{\text {surf }}\left(\nabla \rho_{3}\right)^{2},
\end{aligned}
$$

where $m$ is the nucleon mass and the coefficients $C_{\text {.. }}, D_{\text {.. }}$ are combinations of the Skyrme parameters [44]. The actual Skyrme functional is more complicated than in Eq. (4) as it includes the spin-orbit terms, plus other terms that depend on the spin-orbit densities $\vec{J}$ and are dubbed $J^{2}$ terms. The spin-orbit terms are considered in TDHF and RPA calculations, but they are not included in the semiclassical Vlasov calculations. The $J^{2}$ terms are not included in the TDHF and Vlasov calculations. Although they could be included in RPA, for the sake of comparing it with other models, they are dropped in RPA as well. One may expect that the overall qualitative features of the excitations investigated here are not significantly affected by the approximations we have made. The Coulomb interaction is considered in all frameworks.

We are interested in effects linked to the neutron/proton content of the nuclei under study, thus it is convenient to introduce the symmetry energy per nucleon, $E_{\mathrm{sym}} / A=C(\rho) I^{2}$, where $I=\rho_{3} / \rho$ is the asymmetry parameter and the coefficient $C(\rho)$ can be written as a function of the Skyrme coefficients:

$$
C(\rho)=\frac{\epsilon_{F}}{3}+D_{0} \rho+D_{3} \rho^{\alpha+1}+\frac{2 m}{\hbar^{2}}\left(\frac{C_{\text {eff }}}{3}+D_{\text {eff }}\right) \epsilon_{F} \rho,
$$

with $\epsilon_{F}$ denoting the Fermi energy at density $\rho$.

In the following, we will adopt the recently introduced SAMi-J Skyrme effective interactions [35] based on the fitting protocol of the SAMi interaction [45]. The SAMi-J family has been produced by systematically varying the value of $\mathbf{J}=$ $C\left(\rho_{0}\right)$ (being $\rho_{0}$ the saturation density) from 27 to $35 \mathrm{MeV}$, keeping fixed the optimal value of the incompressibility and effective mass predicted by SAMi and refitting again the parameters for each value of $J$. This produces a set of interactions of similar quality on the isoscalar channel and that, approximately, isolate the effects of modifying the isovector channel in the study of a given observable. The SAMi fitting protocol [45] includes: binding energies and charge radii of some doubly magic nuclei, which allows the SAMi-J family to predict a reasonable saturation density and energy of symmetric nuclear matter (the incompressibility value is $K=245 \mathrm{MeV}$ ); some selected spin-orbit splittings; the spin and spin-isospin Landau Migdal parameters [46]; and, finally, the neutron matter EoS of Ref. [47]. These features allow the
TABLE I. The symmetry energy coefficient at saturation density for the Skyrme interactions employed in our study and the corresponding slope $L$.

\begin{tabular}{lcr}
\hline \hline Interaction & $\mathrm{J}[\mathrm{MeV}]$ & $\mathrm{L}[\mathrm{MeV}]$ \\
\hline SAMi-J27 & 27 & 29.9 \\
SAMi-J31 & 31 & 74.5 \\
SAMi-J35 & 35 & 115.2 \\
\hline \hline
\end{tabular}

new SAMi-J interactions to give a reasonable description of isospin as well as spin-isospin resonances, keeping a good reproduction of well known empirical data such as masses, radii, and important nuclear excitations (see original work for further details).

In our calculations, we employed three SAMi-J parametrizations: SAMi-J27, SAMi-J31 and SAMi-J35 [35]. Since, as mentioned above, the SAMi-J interactions have been fitted to also reproduce the main features of finite nuclei, for the three parametrizations the symmetry energy coefficient gets the same value, $C\left(\rho_{c}\right) \approx 22 \mathrm{MeV}$ at $\rho_{c}=0.6 \rho_{0}$, which would approximately correspond to the average density probed by nuclear masses via the fitting protocol, if one assumes a local density approximation $[36,48]$. The corresponding values of symmetry energy at saturation, together with the values of the slope parameter $L=\left.3 \rho_{0} \frac{d C(\rho)}{d \rho}\right|_{\rho=\rho_{0}}$ are reported in Table I.

\section{B. Numerical details of the calculations and ground-state configuration}

To determine the ground-state configuration of the nuclei under study, different numerical procedures are followed in quantal and semiclassical approaches. In the quantal case, Hartree-Fock (HF) calculations are performed, although two different codes are employed for TDHF and RPA calculations. In the former case, the EV8 code [49] is used, while in the latter case the code SKYRME_RPA [39] is employed. In the present study, we consider selected closed-shell nuclei and some $\mathrm{Sn}$ isotopes known to be spherical (see, e.g., Ref. [50]). In addition, pairing correlations have been neglected to allow for a consistent comparison between the different approaches. Pairing will not play a role in the magic nuclei that we discuss below. In the open-shell spherical systems, pairing is known to affect more the low-lying quadrupole and octupole states than the dipole response [51,52]. More specifically, in Ref. [53] it has been shown that pairing effects have no influence on the dipole polarizability in the ${ }^{116-132} \mathrm{Sn}$ isotopes, especially in the case of SAMi-J31, that is employed here.

In the EV8 code, the HF equations are solved in coordinate space. The mesh size has been taken as $d x=0.8 \mathrm{fm}$. The imaginary time method is adopted, with a fixed time step $d t_{0}=0.36 \mathrm{fm} / \mathrm{c}$. These parameters correspond to standard choices [49]. The total size of the cubic mesh should be large enough to avoid effects of particle evaporation on the TDHF dynamical response. We will consider several choices to test the sensitivity of the results to this parameter. However, the values considered should also ensure a reasonable computational time. 
On the other side, within the fully self-consistent HF+RPA calculations [39] presented here, the ground-state properties of the different nuclei are calculated in coordinate space using box boundary conditions assuming spherical symmetry. Also in this case, we will test different sizes of the box, keeping a radial mesh of $\mathrm{dr}=0.1 \mathrm{fm}$. The same box is used to calculate discrete states at positive energy that are associated with the continuum part of the spectrum. A cutoff energy of $120 \mathrm{MeV}$ (in the single-particle energy) is adopted for the RPA calculations. With this energy cutoff, we have checked that the energy weighted sum rule is satisfactorily fulfilled.

The integration of the Vlasov transport equations is based on the test-particle (t.p.) (or pseudoparticle) method [54], with a number of 1500 t.p. per nucleon in all the cases, ensuring in this way a good spanning of the phase space. The ground-state configuration corresponds to the stationary solution of Eq. (3). Within the Thomas-Fermi (TF) approximation, we adopt the following numerical procedure: neutrons and protons are distributed inside spheres of radii $R_{n}$ and $R_{p}$, respectively. Accordingly, particle momenta are initialized inside Fermi spheres associated with the local neutron or proton densities. Then $R_{n}$ and $R_{p}$ are tuned to minimize the corresponding total energy, associated with the effective interaction adopted in the calculations. Because test particles are often associated with finite width wave packets (we use triangular functions [55]), some surface effects are automatically included in the initialization procedure and in the dynamics, even though explicit surface terms, as those contained in the effective Skyrme interactions, are not considered. This implies that, for the surface terms, one cannot simply use the coefficients associated with the SAMi-J parametrizations. Indeed we observe that a good reproduction of the experimental values of both proton root-mean-square radius and binding energy, for the nuclei selected in our analysis, is obtained when taking $C_{\text {surf }}=D_{\text {surf }}=0$ in our parametrizations. Thus, this choice has been adopted in the following for Vlasov calculations (see Ref. [23] for more details).

\section{Dipole response}

Dipole oscillations and response functions can be investigated, in both TDHF and semiclassical treatments, introducing a small perturbation of the ground-state configuration of the nucleus under study and then looking at its dynamical evolution, as given by Eqs. (1) or (3). Thus, we study the E1 (isoscalar and isovector) response of nuclear systems, considering initial conditions determined by the instantaneous excitation $V_{\text {ext }}=\eta_{k} \delta\left(t-t_{0}\right) \hat{D}_{k}$, along the $z$ direction [42,56]. Here $\hat{D}_{k}$ denotes the operator employed to introduce isoscalar $(k=\mathrm{S})$ or isovector $(k=\mathrm{V})$ dipole excitations and takes the standard form [39]:

$$
\begin{aligned}
& \hat{D}_{S}=\sum_{i=1}^{A}\left(r_{i}^{2}-\frac{5}{3}\left\langle r^{2}\right\rangle\right) z_{i}, \\
& \hat{D}_{V}=\sum_{i=1}^{A}\left[\tau_{i} \frac{N}{A}-\left(1-\tau_{i}\right) \frac{Z}{A}\right] z_{i},
\end{aligned}
$$

where $N$ and $Z$ indicate neutron and proton number, $A=$ $N+Z, \tau_{i}=1(0)$ for protons (neutrons) and $\left\langle r^{2}\right\rangle$ denotes the mean square radius of the nucleus considered. The above definitions [Eqs. (6) and (7)] avoid the undesired effect of the so called spurious state and remove the contribution from the center of mass, respectively. We note that the operator $\hat{D}_{V}$ also contains an isoscalar component, which vanishes only for symmetric $(N=Z)$ systems. According to basic quantum mechanics, if $\left|\Phi_{0}\right\rangle$ is the state before perturbation, then the excited state becomes $\left|\Phi_{k}\left(t_{0}\right)\right\rangle=e^{i \eta_{k} \hat{D}_{k}}\left|\Phi_{0}\right\rangle$. The value of $\eta_{k}$ can be related to the initial expectation value of the collective dipole momentum $\hat{\Pi}_{k}$, which is canonically conjugated to the collective coordinate $\hat{D}_{k}$, i.e., $\left[\hat{D}_{k}, \hat{\Pi}_{k}\right]=i \hbar[57]$.

The same operators defined above are considered in RPA calculations, to extract isoscalar and isovector dipole strength functions: $S_{k}(E)=\sum_{n>0}\left|\left\langle n\left|\hat{D}_{k}\right| 0\right\rangle\right|^{2} \delta\left[E-\left(E_{n}-\right.\right.$ $\left.\left.E_{0}\right)\right]$, where $E_{n}$ is the excitation energy of the state $|n\rangle$ and $E_{0}$ is the energy of the ground state $|0\rangle=\left|\Phi_{0}\right\rangle$.

In TDHF and Vlasov calculations, the strength function is obtained from the imaginary part of the Fourier transform of the time-dependent expectation value of the dipole moment $D_{k}(t)=\left\langle\Phi_{k}(t)\left|\hat{D}_{k}\right| \Phi_{k}(t)\right\rangle$ as

$$
S_{k}(E)=\frac{\operatorname{Im}\left[D_{k}(\omega)\right]}{\pi \eta_{k}},
$$

where $D_{k}(\omega)=\int_{t_{0}}^{t_{\max }} D_{k}(t) e^{i \omega t} d t$, with $E=\hbar \omega$. In these two approaches, we follow the dynamics of the system, looking in particular at the time oscillations of the dipole moments, until $t_{\max }=1800 \mathrm{fm} / \mathrm{c}$. The TDHF equations are solved using the 3D-TDHF code of Refs. [51,58-60], with a time step $d t=0.36 \mathrm{fm} / \mathrm{c}$. A slightly larger time step, $d t=0.50 \mathrm{fm} / \mathrm{c}$, is instead adopted for the solution of the Vlasov equation. As described in Ref. [61], to eliminate the artifacts resulting from a finite time domain analysis of the signal, a filtering procedure was moreover applied by introducing a smooth cutoff function such that

$$
D_{k}(t) \rightarrow D_{k}(t) \exp \left(-\frac{\gamma t}{2 \hbar}\right)
$$

with $\gamma=0.8 \mathrm{MeV}$.

\section{RESULTS}

This section is dedicated to investigating the E1 (IS and IV) response of neutron-rich nuclear systems. To compare with the semiclassical results reported in a previous work [23], we consider in our analysis three closed-shell nuclei: ${ }^{68} \mathrm{Ni}$ (proton closed-shell), ${ }^{132} \mathrm{Sn}$, and ${ }^{208} \mathrm{~Pb}$. Later, to better explore how the features of the dipole response evolve with the neutron/proton content of the nuclei under study, we will also consider two other $\mathrm{Sn}$ isotopes $\left({ }^{100} \mathrm{Sn},{ }^{120} \mathrm{Sn}\right)$ in our analysis.

\section{A. Comparison between quantal and semiclassical approaches}

\section{Ground-state properties and density profiles}

As stressed in the Introduction, we aim at elucidating the role of some global properties, such as density profiles and neutron skin, in determining the main features of the nuclear response. Therefore, as a preliminary step, it is worthwhile 
TABLE II. Neutron and proton root-mean-square radii, their difference, and binding energy for three systems considered in our study, as obtained in TF and HF models with the SAMi-J31 interaction. The experimental values, for charge radius and binding energy, are also indicated [62].

\begin{tabular}{lccccc}
\hline \hline & $\sqrt{\left\langle r^{2}\right\rangle_{n}}[\mathrm{fm}]$ & $\sqrt{\left\langle r^{2}\right\rangle_{p}}[\mathrm{fm}]$ & $\sqrt{\left\langle r^{2}\right\rangle_{n}}-\sqrt{\left\langle r^{2}\right\rangle_{p}}[\mathrm{fm}]$ & $\frac{B}{A}[\mathrm{MeV}]$ \\
\hline & & \multicolumn{4}{c}{${ }^{68} \mathrm{Ni}$} \\
$\mathrm{HF}$ & 4.001 & 3.831 & 0.170 & 8.845 \\
$\mathrm{TF}$ & 4.102 & 3.898 & 0.204 & 9.050 \\
$\mathrm{Exp}$ & - & 3.857 & & - & 8.682 \\
& & & ${ }^{132} \mathrm{Sn}$ & & \\
$\mathrm{HF}$ & 4.927 & 4.664 & & 0.263 & 8.448 \\
$\mathrm{TF}$ & 5.035 & 4.741 & & 0.294 & 8.552 \\
Exp & - & 4.709 & & - & 8.354 \\
& & & ${ }^{208} \mathrm{~Pb}$ & & \\
$\mathrm{HF}$ & 5.654 & 5.456 & & 0.198 & 7.916 \\
$\mathrm{TF}$ & 5.735 & 5.536 & 0.199 & 8.042 \\
Exp & - & 5.501 & - & 7.867 \\
\hline \hline
\end{tabular}

to illustrate the capability of both semiclassical and quantal approaches in reproducing some experimental ground-state quantities. It should be noticed that the HF calculations give an excellent agreement with data if the $J^{2}$ terms are included. Indeed, the SAMi family has been originally fitted including all Skyrme-like terms.

The proton root-mean-square radius and the binding energy evaluated by employing, respectively, a semiclassical treatment in the TF approximation or a self-consistent quantal HF calculation, are listed in Table II, together with the corresponding experimental values. For the sake of completeness, neutron root-mean-square radius and neutron skin thickness are also reported. The SAMi-J31 parametrization of the effective interaction has been employed.

One observes, in both models, a general good reproduction of the experimental values, especially for larger systems, as it should be, according to the mean-field approximation adopted. TF calculations predict a more extended neutron skin as well as slightly larger binding energy values with respect to the HF case. To better emphasize the differences observed between the two approaches, the isoscalar density $\rho$ and the local asymmetry $\rho_{3} / \rho$ profiles are plotted in Figs. 1 and 2, respectively.

With respect to the $\mathrm{HF}$ result, the $\mathrm{TF}$ isoscalar density profile appears flatter in the internal region, especially in the $\mathrm{Ni}$ and $\mathrm{Pb}$ case, indicating a sharper transition from the volume to the surface region. This could be attributed to the numerical treatment of surface effects in Vlasov calculations and to the lack of intrinsic quantal gradient terms, corresponding to the $\hbar^{2}$ terms in the Wigner-Kirkwood $\hbar$ expansion of the distribution function $[63,64]$. One expects that these differences will affect the details of the modes mostly involving surface oscillations. Looking at Fig. 2, one observes some differences between quantal and semiclassical predictions also in the isovector density $\rho_{3}$. Semiclassical calculations are characterized by a larger neutron drift towards the surface. Some differences appear also in the more internal region, evidencing the role of shell effects in shaping the fine details of the nuclear structure.

\section{Dipole response and strength function}

Next, we investigate the dipole response. Figure 3 shows the time evolution of IS and IV dipole moments in the system ${ }^{132} \mathrm{Sn}$, as obtained by using an initial IS or IV perturbation. In our analysis we choose, as perturbation strength, the following values: $\eta_{S}=1.0 \times 10^{-4} \mathrm{fm}^{-3}, \eta_{V}=1.0 \times 10^{-4} \mathrm{fm}^{-1}$ in TDHF calculations and $\eta_{S}=2.5 \times 10^{-3} \mathrm{fm}^{-3}, \eta_{V}=1.3 \times$ $10^{-1} \mathrm{fm}^{-1}$ for Vlasov ones, respectively. The numerical procedure adopted to solve the Vlasov equation, related to the use of a finite number of test particles to map the one-body distribution function, introduces some numerical noise, implying to consider larger amplitude perturbations, with respect to TDHF. One may generally note larger damping effects in the Vlasov calculations, probably related to the finite number of test particles and to the larger amplitude of the initial per-

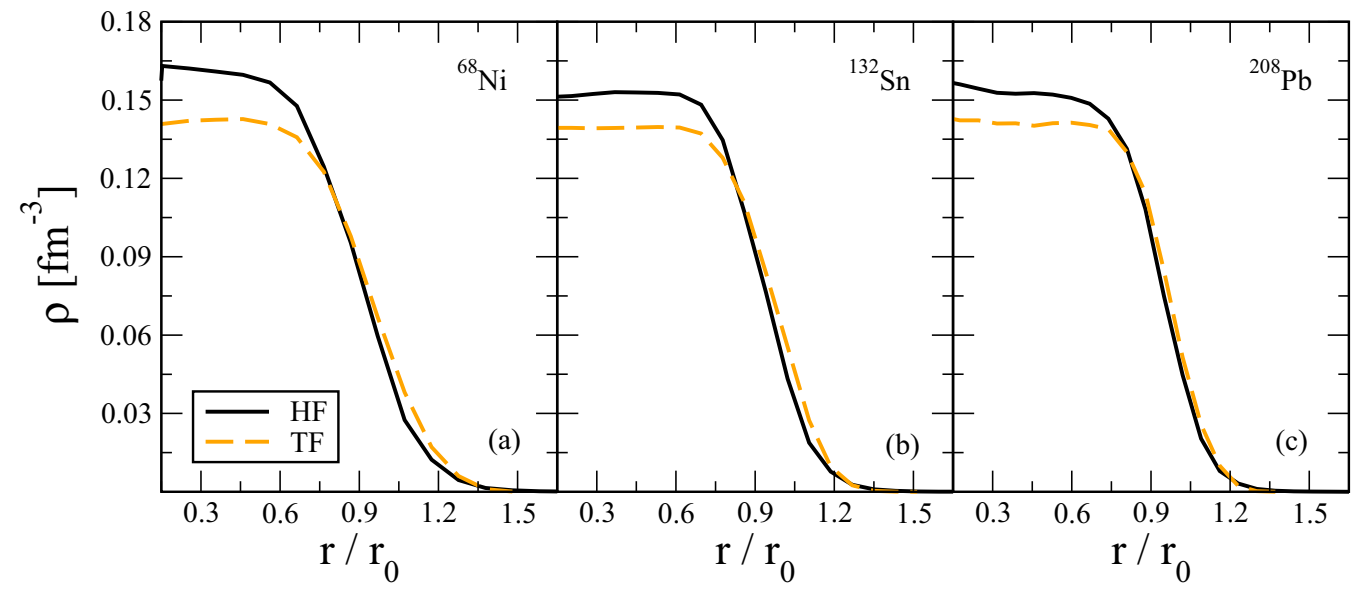

FIG. 1. The isoscalar density profiles for ${ }^{68} \mathrm{Ni},{ }^{132} \mathrm{Sn}$, and ${ }^{208} \mathrm{~Pb}$, from Hartree-Fock and Thomas-Fermi models, versus the normalized radius $r / r_{0}$, with $r_{0}=1.2 A^{1 / 3}$. 


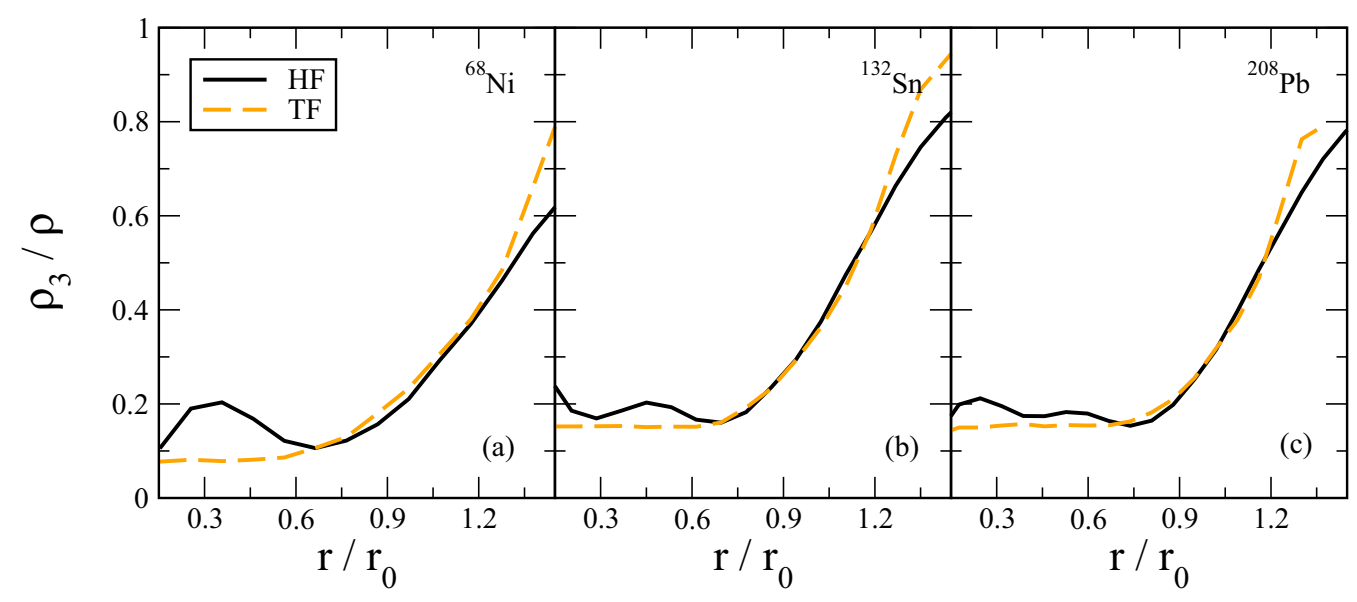

FIG. 2. The local asymmetry profile for ${ }^{68} \mathrm{Ni},{ }^{132} \mathrm{Sn}$, and ${ }^{208} \mathrm{~Pb}$, from Hartree-Fock and Thomas-Fermi models, versus the normalized radius $r / r_{0}$, with $r_{0}=1.2 A^{1 / 3}$.

turbation, that may induce nonlinear effects, i.e., the coupling to other multipoles, and increase particle evaporation.

Figure 4 displays the corresponding IV and IS dipole strength functions in ${ }^{68} \mathrm{Ni},{ }^{132} \mathrm{Sn}$, and ${ }^{208} \mathrm{~Pb}$. Looking at the bottom panels, one notices that the IV dipole strength is clearly dominated by the collective IV GDR mode peaked in the energy region around 12-16 MeV. Despite the differences observed between the semiclassical and quantal ground-state features, the IV dipole strength deduced within the TDHF model is generally well reproduced by the corresponding Vlasov calculation. The agreement is particularly satisfying for the energy of the main IV peak, especially when larger systems are considered. Some strength is observed at low energy, i.e., in the region of the PDS, albeit the corresponding peaks

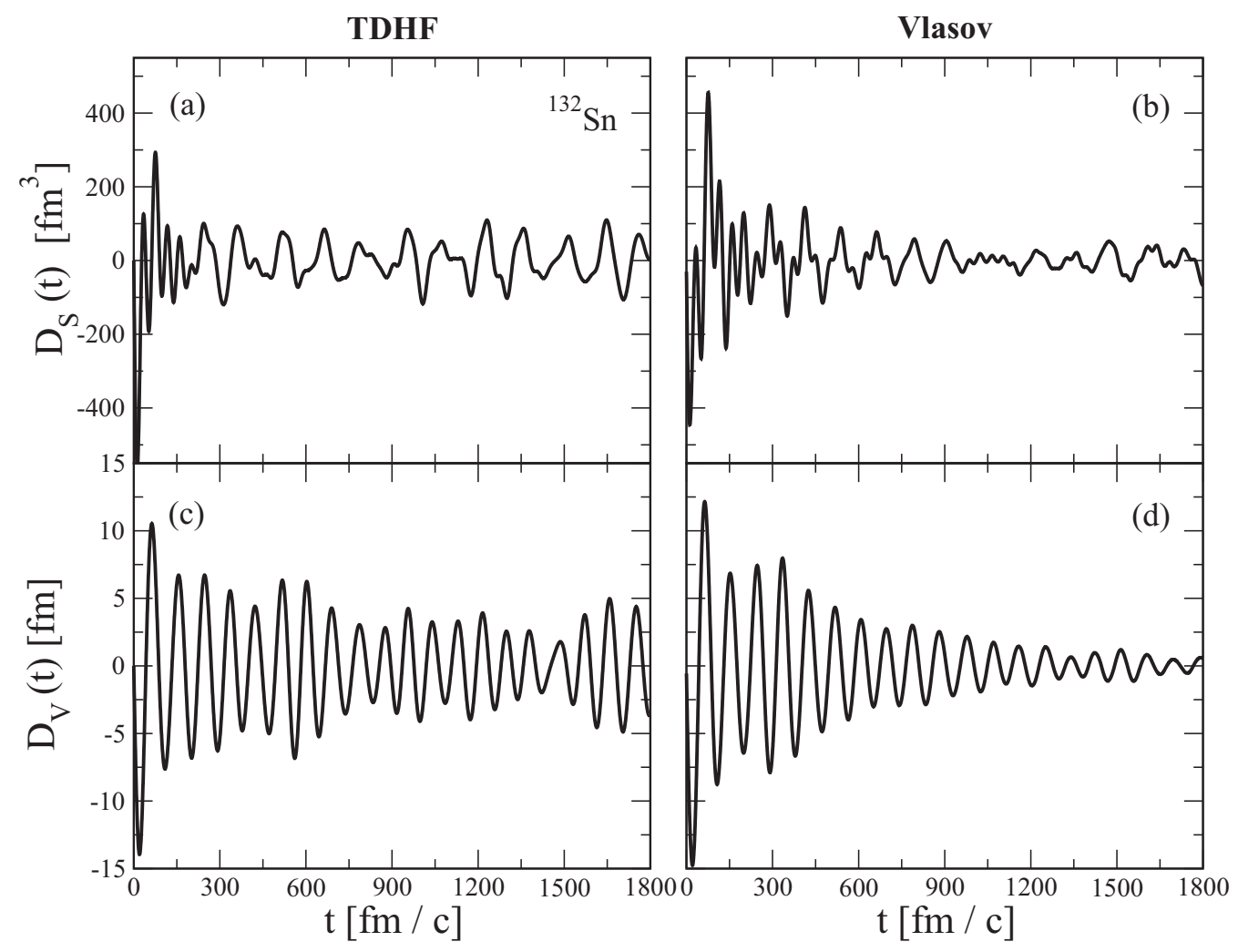

FIG. 3. (Top panels) The time evolution of the IS dipole moment for ${ }^{132} \mathrm{Sn}$ and SAMi-J31 interaction, as obtained in TDHF (left panel) and Vlasov (right panel) calculations. (Bottom panels) The same as the top panels, but for the IV dipole moment. TDHF dipole moments have been rescaled to the Vlasov perturbation strength. 

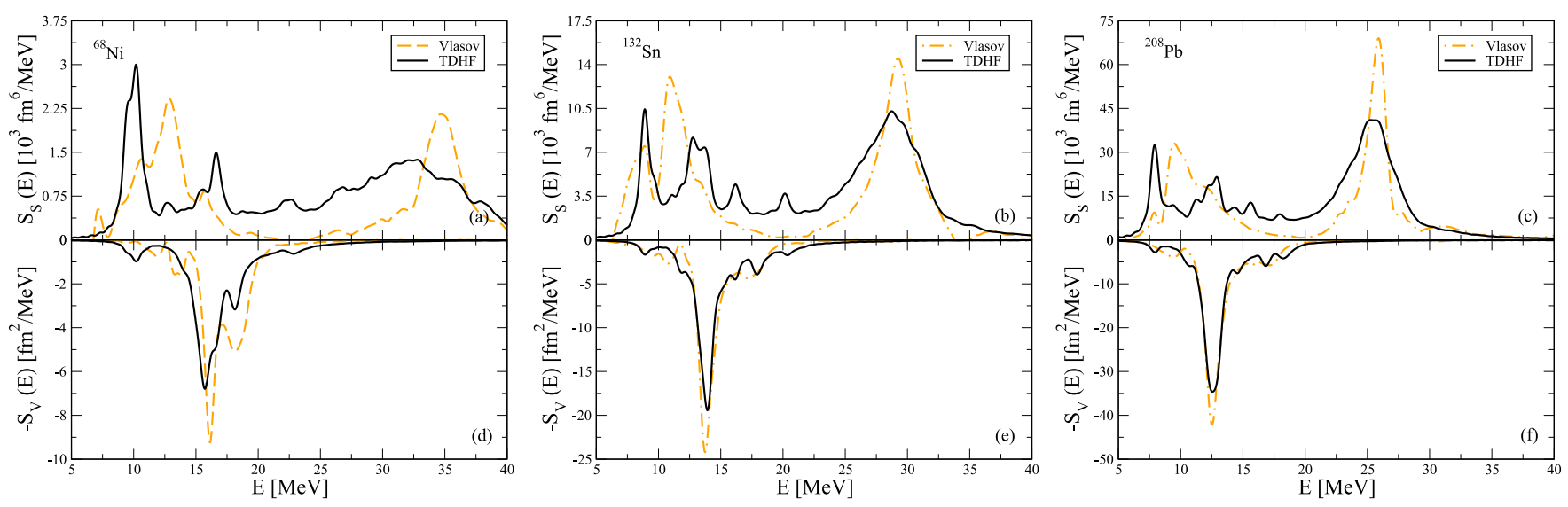

FIG. 4. (Top panels) The strength function of the IS response of the closed shell nuclei ${ }^{68} \mathrm{Ni},{ }^{132} \mathrm{Sn}$, and ${ }^{208} \mathrm{~Pb}$, with $\mathrm{SAMi}-\mathrm{J} 31$ interaction. Full lines represent the results from TDHF calculations, dashed lines the ones from Vlasov calculations. (Bottom panels) Similar to the figures in the top panels, but for the strength function of the IV response.

look shifted in Vlasov calculations, with respect to TDHF. Though it is interesting to notice that the PDS also emerges in semiclassical calculations, we stress that the details of this low-energy IV contribution, namely its degree of collectivity and precise energy location, are strongly affected by shell effects and by the ingredients of the residual interaction, as pointed out in recent investigations $[65,66]$.

Concerning the IS dipole strength (top panels in Fig. 4), the scenario is more complicated. In the case of the large systems, the two models give close values for the centroid of the high-energy peak, in the excitation energy domain of the IS GDR (around $30 \mathrm{MeV}$ ), where the compressional modes dominate [67]. For ${ }^{68} \mathrm{Ni}$, a shift to higher energy is clearly evidenced in the Vlasov case, with respect to TDHF. Moreover, TDHF calculations lead to a larger fragmentation of the strength function, particularly in the $\mathrm{Ni}$ case.

These differences may arise from the fact that in Vlasov simulations the evaporated particles are more abundant and may leave the calculation box. A remarkable discrepancy can be identified in the shape of the nuclear response in the low-energy regime. Two main regions of contribution can be recognized, which are well separated in energy in the TDHF case (see for instance the contributions around 8 and $13 \mathrm{MeV}$ in the ${ }^{132} \mathrm{Sn}$ case). In the same energy region, the Vlasov calculations show two main peaks which are closer in energy (around 8 and $11 \mathrm{MeV}$ for ${ }^{132} \mathrm{Sn}$ ) and not always clearly distinguishable (see in particular the result for ${ }^{208} \mathrm{~Pb}$ ). Moreover, the relative weight of the two peaks is different in TDHF and Vlasov calculations.

It is worthwhile to notice that in previous semiclassical studies [41], where isoscalar toroidal excitations were investigated, the modes emerging in this energy region have been associated with surface oscillations. In particular, the lowest energy one corresponds to oscillations deeply involving the outer surface zone, whereas the second mode (of higher energy) would correspond to standard toroidal dipole excitations, associated with the oscillation of the surface against the bulk region [41].

As anticipated above, the energy position predicted for these isoscalar surface peaks is quite different in Vlasov and
TDHF calculations. In particular, in TDHF calculations, the second region of considerable strength is shifted to higher frequency. This discrepancy could originate from the approximations done in the semiclassical approach, like the lack of gradient terms and, however, from the numerical procedure adopted to treat surface effects in this case, as already noted for the ground-state configuration. Since isoscalar gradient terms give a positive contribution to the restoring force, we may expect higher oscillation frequencies in TDHF. Last but not least, the details of the low-lying excitations are very likely to be also affected by shell effects.

Moreover, TDHF calculations seem to favor the lowest energy peak, whereas the opposite happens in the Vlasov results. This latter behavior could be connected to the different density profile predicted by the two calculations. A sharper evolution from the bulk to the surface region, as observed in the Vlasov case, seems to favor the dominance of the standard toroidal mode. However, a smoother density profile enhances surface effects, leading to more robust oscillations in the lowestenergy region. A further insight about the volume/surface nature of these excitation is gained, however, by looking at the shape of the corresponding transition densities, as discussed in the following (see Sec. III D).

\section{B. Isoscalar-isovector mixing in n-rich systems}

As stated in the Introduction, one of the goals of our analysis is to get a deeper insight into the isoscalar-isovector mixing which characterizes the excitation modes of nuclei with an unbalanced number of protons and neutrons. Let us consider TDHF calculations for the system ${ }^{132} \mathrm{Sn}$, with the SAMi-J31 effective interaction. Fig. 5 (left panels) shows that it is possible to extract a sizable IS response by perturbing the nucleus not only with an initial IS excitation (top), but also by employing an initial IV excitation (bottom). Similarly, the investigation of the IV response carried out by employing the two kinds of initial perturbation (see Fig. 6, left panels) shows that IS excitations also generate an IV strength. In each panel of Figs. 5 and 6, the relative height of the peaks will depend 


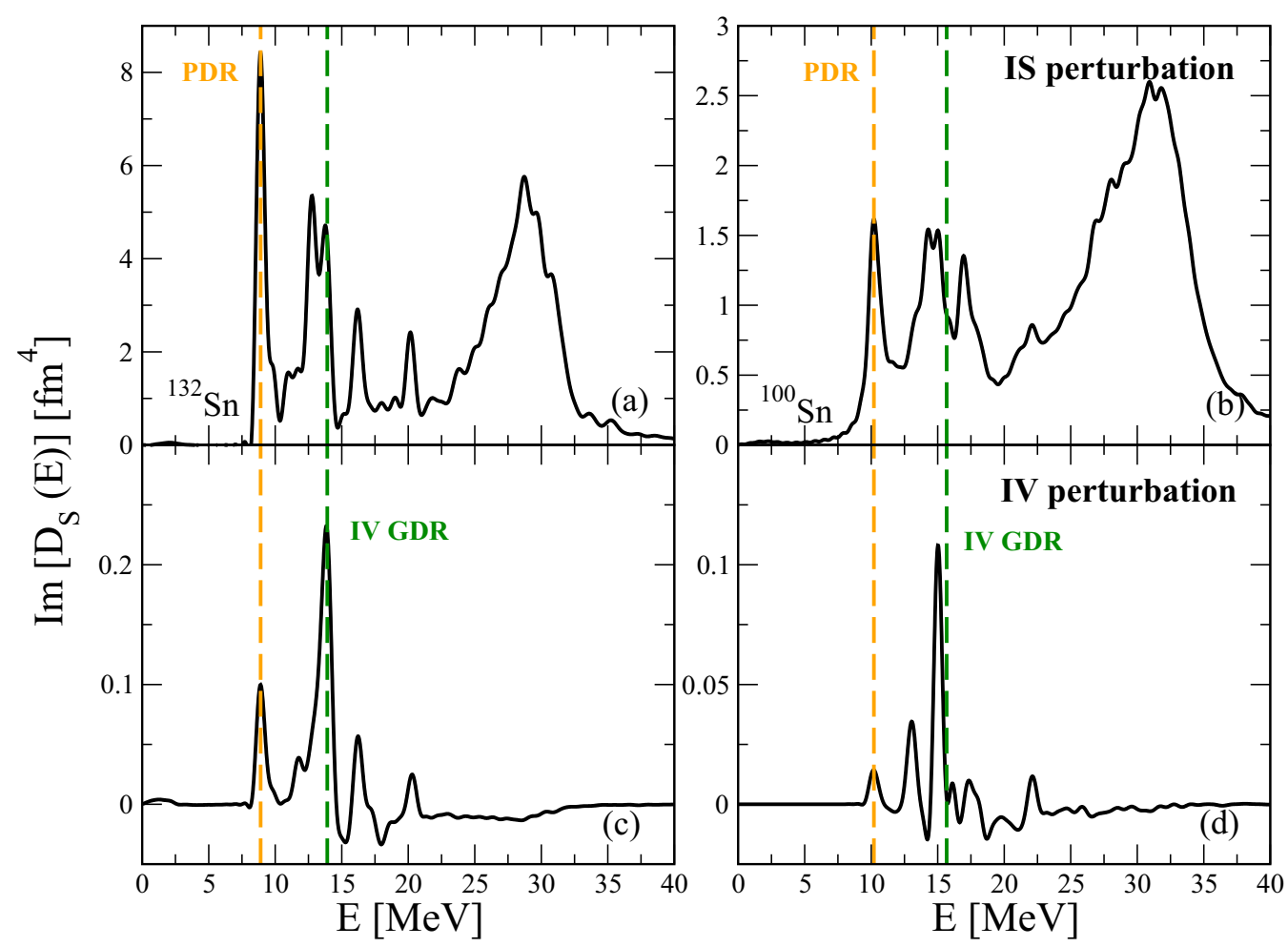

FIG. 5. The imaginary part of the IS response with IS perturbation (top) and IV perturbation (bottom), respectively, as obtained for ${ }^{132} \mathrm{Sn}$ (left panels) and ${ }^{100} \mathrm{Sn}$ (right panels) with SAMi-J31 in TDHF calculations.

on the initial perturbation type and on the intrinsic structure of the mode considered.

This characterization holds for almost all the main modes, which are excited by both the IS and IV perturbations, according to their mixed nature. Looking at the strength of the peaks in the different panels, it appears that, whereas the IV GDR manifests its well established predominant isovector nature, though with some mixing, the lowest-energy excitation (indicated as PDR) turns out to be mostly an isoscalarlike mode (see also the analysis in Ref. [66]), which, however, can be excited also by an IV perturbation, owing to the coupling existing between isoscalar and isovector modes in asymmetric systems. Only for the IS GDR, the IV projection comes out to be negligible, thus indicating a quite robust isoscalar nature of this mode. Looking at the bottom panels of Figs. 5 and 6 , one can notice, as a quite interesting detail, that the two mixed projections, i.e., the IS(IV) response generated by an IV(IS) perturbation have the same structure. This confirms the consistency of our calculations. It should be noticed that similar results about the isoscalar-isovector mixing of the excitation modes in neutron-rich systems have been obtained also in semiclassical calculations [23].

To better clarify the role of the isospin asymmetry in shaping the mixing observed in the dipole response, we extended our analysis to the nuclear system ${ }^{100} \mathrm{Sn}$, that is the doublemagic nucleus of the $\mathrm{Sn}$ isotope chain, which is constituted by an equal number of protons and neutrons.

According to the framework depicted above, one expects the coupling between the modes excited by the two perturbation kinds to be quenched in this case. Indeed, this is in line with the results plotted in the right panels of Figs. 5 and 6. Beyond dispute, in this case, the correspondence in the dipole strengths associated with the two perturbations considered is reduced. For instance, the PDS is not observed, i.e., there is no IV strength in the PDR region in the case of IV excitations (top right panel of Fig. 6). This stresses once again the prominant isoscalar nature of the pygmy mode and the strong connection of its IV counterpart with the neutron richness of the nucleus considered. In the same way, owing to its isovector nature, the IV GDR peak (see the dashed green line in the right panels of Figs. 5 and 6) has a reduced strength in the IS response obtained with an IS perturbation (top right panel of Fig. 5). However, even for the symmetric ${ }^{100} \mathrm{Sn}$, the cross responses (right bottom panels) evidence the presence of some IS-IV mixing, mainly for the modes located in the region between the PDR and the IV GDR. In particular, a noticeable degree of mixing is observed just slightly below the IV GDR, reflecting a sudden transition from IS to IV excitations. Therefore, even though the scenario for ${ }^{100} \mathrm{Sn}$ is partially simplified by its $N=Z$ nature, the general picture has not a trivial interpretation overall. The mixing observed arises from the Coulomb interaction, which breaks the symmetry between neutron and proton response. For the following analyses we will concentrate only on the IV (IS) response induced by an IV (IS) perturbation.

\section{Sensitivity to the effective interaction}

To discuss the impact of the employed effective interaction on the dipole response, we show in Fig. 7 the results obtained 

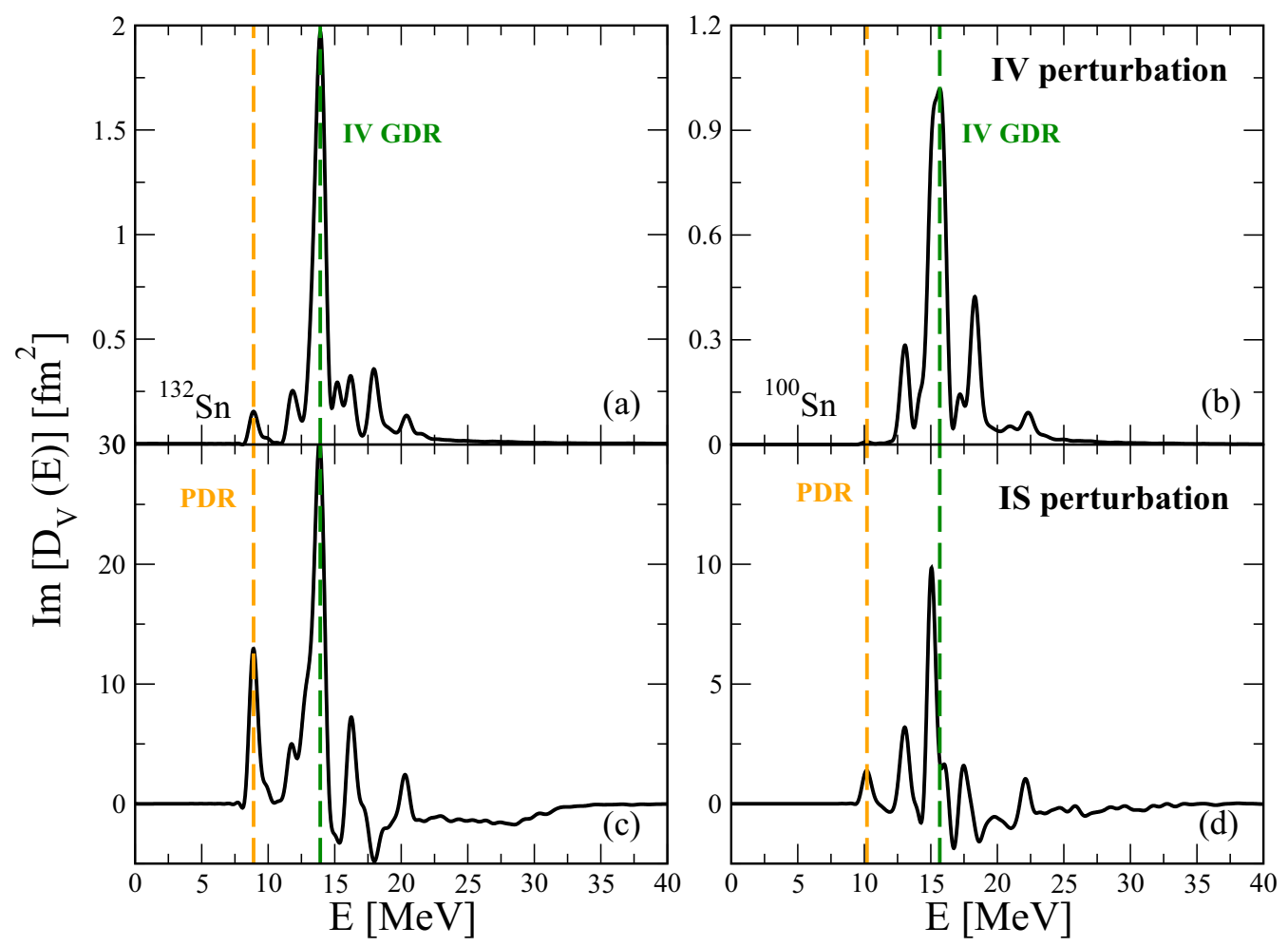

FIG. 6. The imaginary part of the IV response with IV perturbation (top) and IS perturbation (bottom), respectively, as obtained for ${ }^{132} \mathrm{Sn}$ (left panels) and ${ }^{100} \mathrm{Sn}$ (righ panels) with SAMi-J31 in TDHF calculations.

for ${ }^{132} \mathrm{Sn}$, using three SAMi-J parametrizations differing by the (J-L) combination values (see Table I). It is well known $[68,69]$ that the IV response is quite sensitive to the symmetry

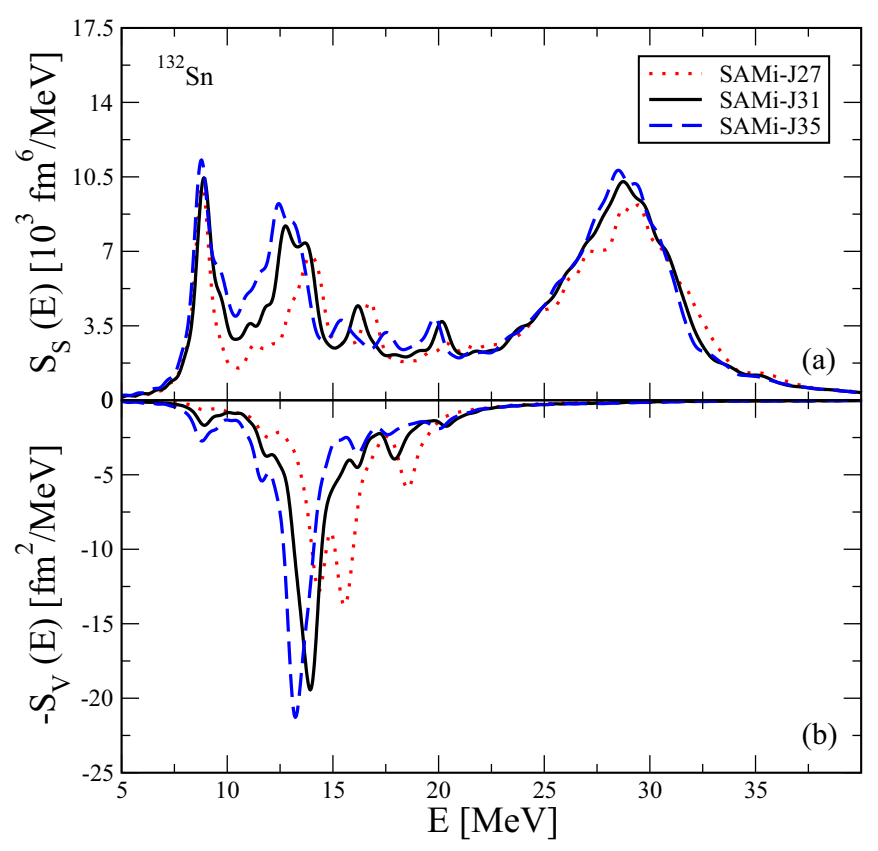

FIG. 7. The IS (a) and IV (b) dipole response for ${ }^{132} \mathrm{Sn}$, as obtained in TDHF calculations when employing three SAMi-J parametrizations. energy details, as we also observe here. In particular, the strength in the region below the IV GDR increases with L $[66,70]$. We notice that, within the adopted interactions, also the neutron skin thickness increases with L (see Tables I and II). Also the frequency of the IV GDR is affected, and it moves to higher values as $\mathrm{L}$ decreases, reflecting the larger value of the symmetry energy at low density (below $\rho_{c}$ ). A splitting of the resonance in two peaks occurs in the case of the SAMi-J27 interaction.

However, we observe only a slight sensitivity of the IS response to the interactions considered in our study. The shift observed for the second relevant IS peak is probably related to the different isoscalar surface terms of the SAMi-J interactions considered, whose strength decreases from J27 to J35. This observation supports the important impact of surface terms on the features of this mode, as already discussed when comparing TDHF and Vlasov results. The compressional IS GDR is insensitive to the choice adopted for the interaction, as one would expect considering the SAMi-J parametrizations are characterized by the same compressibility value.

\section{Comparison between TDHF and RPA}

In this section, we aim at undertaking a detailed comparison of the dipole response which is extracted within the two quantal approaches employed in our work: TDHF and RPA. The two models are equivalent from the theoretical point of view, at least in the limit of small oscillations, so this analysis allows one to highlight possible spurious effects introduced by the technical procedure adopted and therefore isolate only the 


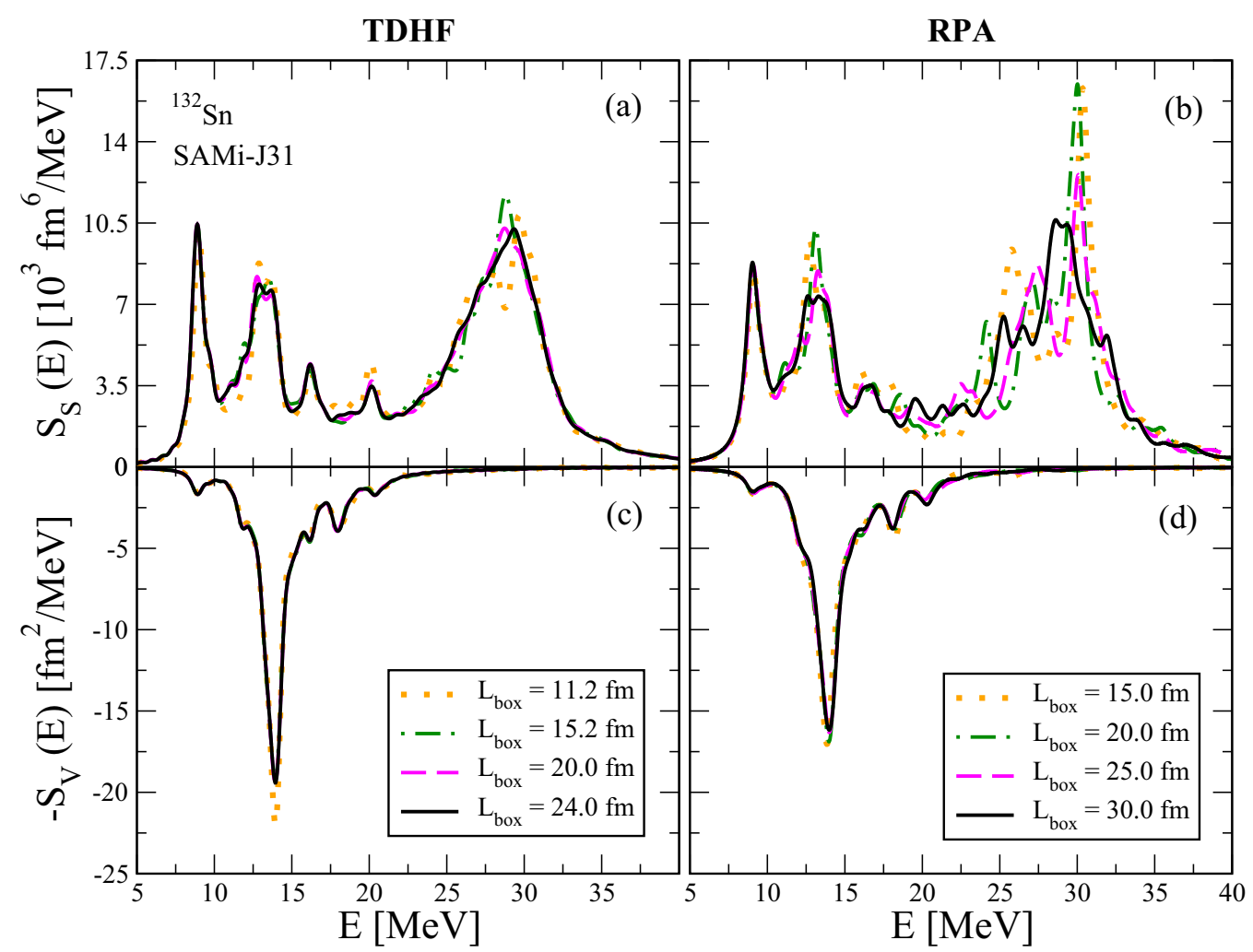

FIG. 8. The strength function of the IS (top panels) and IV (bottom panels) response as obtained in TDHF (left panels) or in RPA calculations (right panels) for ${ }^{132} \mathrm{Sn}$ with SAMi-J31 interaction and different box sizes.

relevant physical features. Moreover, this study helps in giving some hints concerning the numerical parameters to be adopted to ensure the best possible agreement between the two codes, which could be used as a reference also for future works.

For this analysis, we discuss the results obtained for the ${ }^{132} \mathrm{Sn}$ system, employing the SAMi-J31 interaction. Figure 8 presents a comparison of the IS and IV dipole response, as obtained in TDHF calculations, with corresponding RPA calculations. In the latter, the strength function is calculated by convoluting the transition probability with a Lorentzian function of width equal to $0.5 \mathrm{MeV}$. However, in the TDHF results, the spread originates from the finite time interval considered to follow the dynamics and from our smoothing procedure (see Sec. II C).

For the two approaches, we have explored the dependence of the results on the size of the box considered. This ingredient determines the discretization of the single-particle states in the continuum, so that it could affect the details of the oscillation modes at higher frequency. We denote by $L_{\text {box }}$ either half of the side of the cubic box employed in THDF calculations or the radius of the spherical box considered in RPA calculations. Within the spanned range, the values obtained for both the IS and IV Energy Weighted Sum Rule (EWSR) are convergent and consistent between THDF and RPA calculations (within $0.1 \%$ ).

As one can see in Fig. 8, the IV response is nearly insensitive to $L_{\text {box }}$ (within the range considered) and an excellent agreement is obtained between the two approaches. The exci- tation energies of the modes characterizing the IV response are indeed lower, and the coupling with the continuum is smaller than in the case of the IS response. As far as the IS component is concerned, the TDHF response is slightly affected by the $L_{\text {box }}$ parameter and practically converges to its final shape already for $L_{\text {box }}=20 \mathrm{fm}$. The RPA calculations exhibit, within a similar range of values as adopted in the TDHF case, a larger sensitivity to $L_{\text {box }}$ in the high-energy region of the IS spectrum. At present, although we cannot prove it, we could say that the differences between TDHF and RPA may be simply due to different discretization procedures.

An indication along this line can be seen in Fig. 9, that shows a comparison between $\operatorname{TDHF}\left(L_{\mathrm{box}}=20 \mathrm{fm}\right)$ and RPA $\left(L_{\text {box }}=30 \mathrm{fm}\right)$. Here, at variance with Fig. 8 , the RPA strength has been convoluted with a Lorentzian function of energy-dependent width

$$
\Gamma(E)= \begin{cases}e^{-\frac{(E-30)^{2}}{25^{2}} \ln 2} & 5 \leqslant E \leqslant 55 \mathrm{MeV} \\ 0.5 & \text { elsewhere }\end{cases}
$$

which leads to a maximum width of $1.0 \mathrm{MeV}$ in the energy region of the IS GDR.

One can conclude that, in spite of the different degree of sensitivity to some technical ingredients, such as the box size, a very good agreement is observed between TDHF and RPA calculations, as far as the IS and IV dipole responses are concerned. 


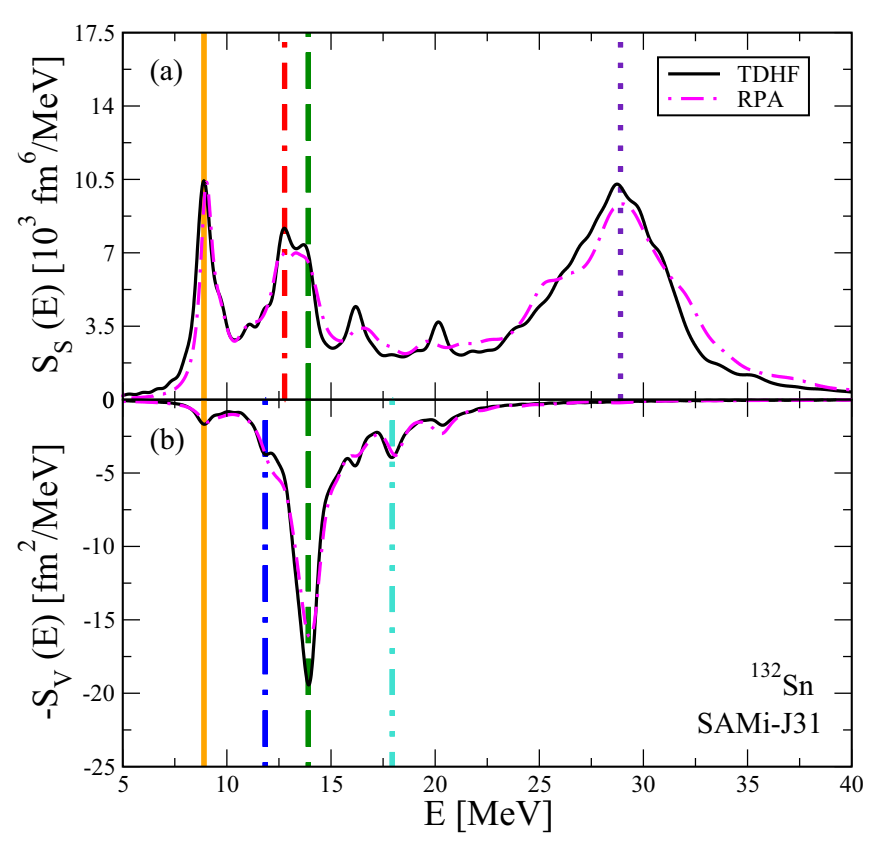

FIG. 9. The strength function of the IS (a) and IV (b) response for ${ }^{132} \mathrm{Sn}$ with SAMi-J31 as obtained in TDHF or in RPA calculation, with $L_{\mathrm{box}}=20$ or $30 \mathrm{fm}$, respectively. The vertical lines indicate the energy of the modes selected for the transition density analysis (see Sec. III E).

\section{E. Transition densities}

In addition to the investigation of the dipole strength discussed so far, the analysis of the transition densities associated with the different excitation modes of the system is very instructive since it delivers important information about the spatial structure related to the dynamics of every excitation. To undertake this analysis in TDHF and Vlasov calculations, we need to evaluate the local spatial density as a function of time. To reduce numerical fluctuations, we take into account the cylindrical symmetry of the initial perturbation and, averaging over the azimuthal $\phi$ angle, we extract the density $\rho_{q}(r, \cos \theta, t)$ and the corresponding fluctuation $\delta \rho_{q}(r, \cos \theta, t)=\rho_{q}(r, \cos \theta, t)-\rho_{q}\left(r, t_{0}\right)$, where $\cos \theta=z / r$ and $\rho_{q}\left(r, t_{0}\right)$ denotes the ground-state density profile, which only depends on $r$. As suggested in Ref. [41], assuming that the amplitude of the oscillation is weak (linear response regime), the spherical symmetry of the ground state and the dipole shape of the excitation operator imply that the transition density can be written, at each time, as $\delta \rho_{q}(r, \cos \theta, t)=\delta \rho_{q}(r, t) \cos \theta$. Then one can finally extract the transition density just as a function of the radial distance $r$, by averaging over the polar angle the quantity $\delta \rho_{q}(r, t)$.

It is clear that, both in Vlasov and TDHF calculations, the perturbation $\mathrm{V}_{\text {ext }}$, at $t=t_{0}$, induces simultaneously all modes which can be excited by the operator $\hat{D}_{k}$. Thus the corresponding density oscillations observed along the dynamical evolution will appear as the result of the combination of the different excitation modes. To pin down the contribution of a given mode, of energy $E$, to the density oscillations, one can compute the Fourier transform of $\delta \rho_{q}(r, t)$ :

$$
\delta \rho_{q}(r, E) \propto \int_{t_{0}}^{\infty} d t \delta \rho_{q}(r, t) \sin \frac{E t}{\hbar} .
$$

In practice, since the simulation runs only to $t_{\max }=$ $1800 \mathrm{fm} / \mathrm{c}$, the sine function is multiplied by a damping factor, as in the strength function $S_{k}(\mathrm{E})$.

We notice that, in RPA calculations, one does not need to use any auxiliary prescription, since the transition densities are directly evaluated from the forward and backward amplitudes solution of the RPA matrix, associated with a given energy eigenvalue $E$ [see Eqs. (36) and (37) in Ref. [39]]. Nonetheless, in principle, it could be possible to average the RPA transition densities in a given energy window.

It is well known that, in symmetric matter, neutrons and protons oscillate with exactly equal (isoscalar) or opposite (isovector) amplitudes. In neutron-rich systems, the picture is more complex; however, one can still identify isoscalarlike modes, when the two nuclear species oscillate in phase, and isovectorlike modes, with neutrons and protons oscillating out of phase. Apart from this information, connected to the mixed character of each mode, the overall spatial structure of the transition densities tells us which part of the system (internal part or surface) is more involved in the oscillation.

In dynamical calculations, dipole excitations are directly excited by a given (IS or IV) perturbation. Hence IS(IV)like oscillations, and corresponding transition densities, are better identified when an initial IS(IV) perturbation is applied. Actually, this possibility to directly probe the response of the system to specific excitations could also help to disentangle between modes having similar energies but different nature. However, the modes with a strong IS-IV mixing react to both (IS and IV) excitations, so the associated transition density can be extracted from both kinds of calculations.

Here we present the transition densities related to the modes giving a sizeable contribution to the IS dipole strength function (Fig. 10) and/or to the IV one (Fig. 11), as obtained for the system ${ }^{132} \mathrm{Sn}$ in TDHF, Vlasov and RPA calculations. For the Vlasov calculations, we consider the same modes identified in Ref. [23]. The energies considered in TDHF and RPA calculations are indicated by vertical bars in Fig. 9.

As a general feature, it should be noticed that TDHF and RPA calculations lead to very similar results. The first row of the two figures displays the structure of what we may call PDR (full orange bar in Fig. 9), which manifests itself as an isoscalarlike mode, but with also an isovector contribution. Indeed, in TDHF and Vlasov calculations, essentially the same structure is observed when the transition density is extracted from IS or IV perturbations, though with a reduced amplitude in the latter case.

The structure obtained in quantal calculations is in agreement with previous results [66] and is qualitatively well reproduced also by the semiclassical density oscillations, except for the behavior in the central region which could be related to the trend observed in the quantal isovector density profiles (see Fig. 2). One can see that density oscillations involve deeply the surface region (see the behavior for $r$ between 5 and $9 \mathrm{fm}$ ). This is in line with the observation that this mode 


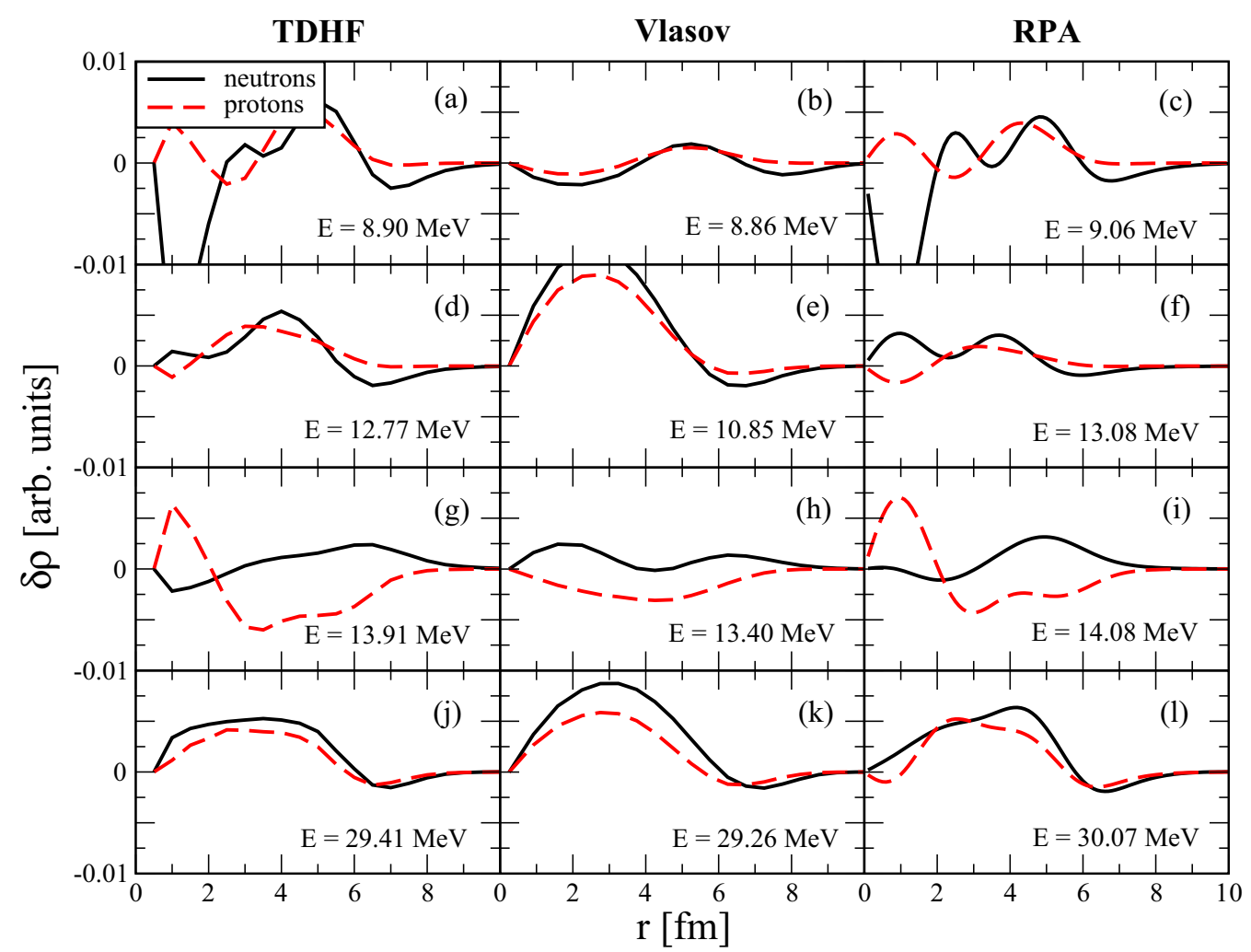

FIG. 10. The transition densities related to the main peaks evidenced in Fig. 9, as obtained in the IS strength function for ${ }^{132} \mathrm{Sn}, \mathrm{SAMi}-\mathrm{J} 31$ parametrization and for the three different models considered. Corresponding energies are also indicated.

is particularly robust in nuclei exhibiting a diffuse density profile, as discussed in Sec. III A.

In the low-lying energy domain (below the IV GDR), a second peak is observed both in the IS and IV dipole strength (dash-dotted blue and dash-dash-dotted red bars in Fig. 9, respectively). These peaks reflect two distinct excitation modes, though their energy is close. The corresponding transition densities are displayed in the second row of Figs. 10 and 11 and, as it is particularly clear in the Vlasov case, manifest their isoscalarlike or isovectorlike nature, respectively. This result indicates that, in addition to the PDR, it is possible to recognize at least other two distinct modes, with different structure, in the energy region below the IV GDR. As compared to the PDR, for this second isoscalarlike mode (compare first and second rows of Fig. 10) density oscillations look shifted to the left, thus involving more the internal part of the system. Actually, this mode should correspond to standard toroidal excitations, where the surface moves against the core. Thus, we expect this mode to be more robust in nuclei with a sharper evolution from the volume to the surface in the density profile.

A deeper investigation on the nature of the modes lying in this low-energy domain will be tackled in Sec. III F.

The structure of the IV GDR (dashed green bar in Fig. 9) is plotted in the third rows of the two figures. In all the cases, the well-established isovectorlike structure corresponding to the semiclassical Goldhaber-Teller (GT) picture is well represented, with essentially one prominent oscillation, having a maximum close to the nuclear surface (see Fig. 11)
[71]. The mode presents also a sizeable isoscalar component; indeed quite similar transition densities, though of reduced amplitude, are extracted considering an initial IS perturbation (Fig. 10) [72].

Lastly, the last rows are dedicated to display the structure of two volume modes: the IS GDR peak obtained in the high-energy region of the IS response (Fig. 10, dotted violet bar in Fig. 9) and the isovector-like peak emerging in the IV response beyond the IV GDR (Fig. 11, dash-dot-dotted cyan bar in Fig. 9). One can notice that the latter IV peak exhibits a structure which is typical of the Steinwedel-Jensen (SJ) description, characterized by a kind of double oscillation and deeply involving also the internal part of the system. The three models compare very well in this case.

To conclude, from this analysis it emerges that semiclassical calculations are able to grasp the main features of the density oscillations associated with the excitation modes considered here, though volume modes are described better than the ones characterized by important surface contributions.

\section{F. Low-lying energy modes for Sn isotopes}

We focus here on low-energy modes, which are more intriguing and controversial, exploring how their features evolve with the isospin asymmetry content of the systems. A large amount of investigations has been devoted in recent years to the behavior of a variety of isotopes, from light to heavy, from spherical to deformed, and from normal to superfluid nuclei, to shed light on the properties of the PDR [73]. Although we 


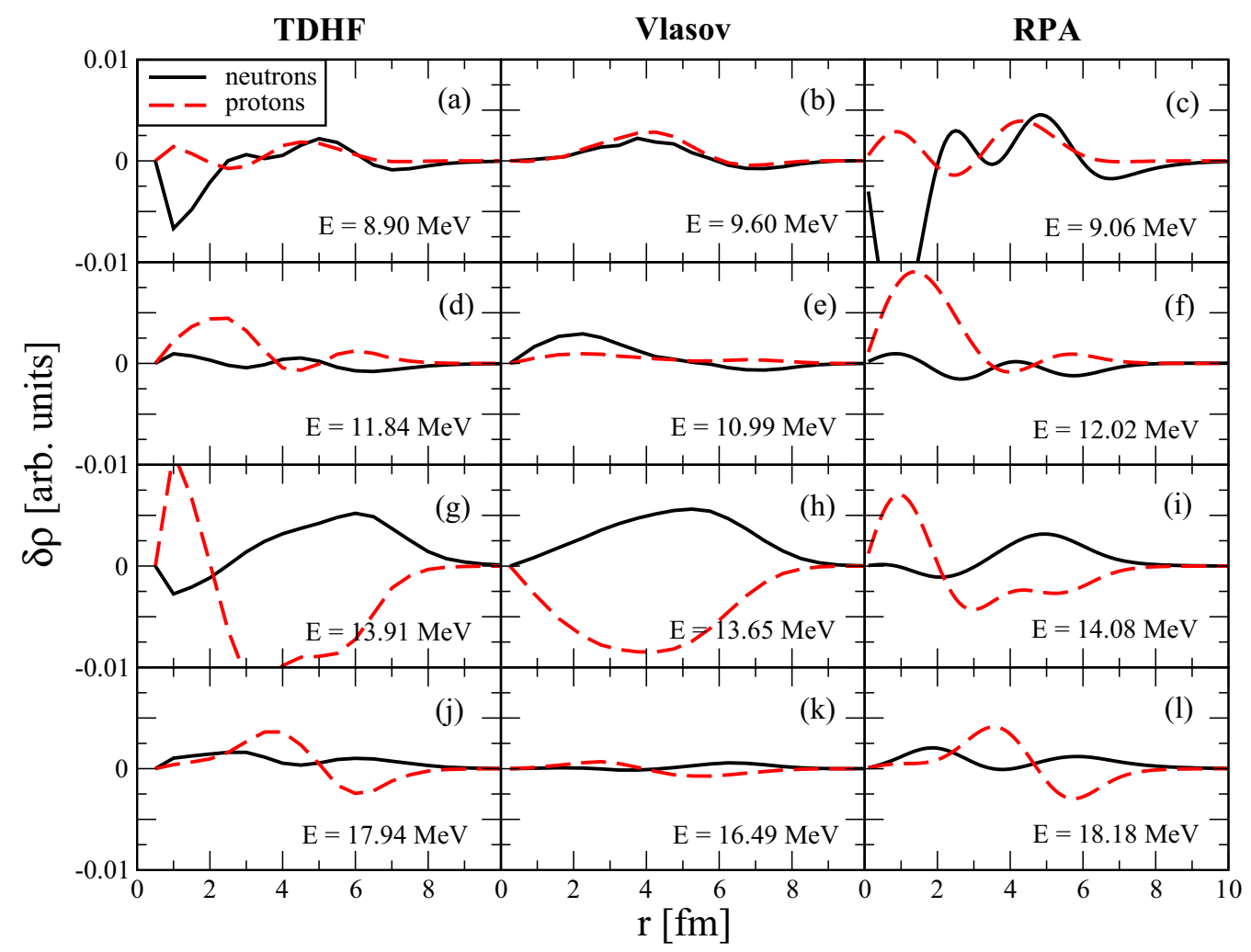

FIG. 11. The transition densities related to the main peaks evidenced in Fig. 9, as obtained in the IV strength function for ${ }^{132}$ Sn, SAMi-J31 parametrization and for the three different models considered. Corresponding energies are also indicated.

are not going to develop here a systematic study in the strict sense, with the aim of elucidating our understanding of the structure of the low-lying energy modes, in this section we are looking at the properties of these excitations in three spherical nuclei belonging to the $\mathrm{Sn}$ isotope chain: the semi-magic nucleus ${ }^{120} \mathrm{Sn}$ and two double magic-nuclei, namely, ${ }^{100} \mathrm{Sn}$ and ${ }^{132} \mathrm{Sn}$. The latter $\mathrm{Sn}$ isotope is the one already considered in the previous sections. In such a way, we can isolate the effect of the $N / Z$ ratio on the isoscalar-isovector mixing and on the structure of the modes we wish to analyze.
In analogy with the investigation carried on for the closed shell nuclei examined in the first section, in Figs. 12-14 the isoscalar density and local asymmetry profiles are plotted, for the three Sn isotopes considered. The three SAMi-J parametrizations of the effective interaction introduced above are adopted: SAMi-J27, SAMi-J31, SAMi-J35. In such a way, it will also be possible to probe the effects of modifying the isovector channel of the functional considered on the observables under study. To better compare the structure of these profiles, in these figures, as in Figs. 1 and 2, we

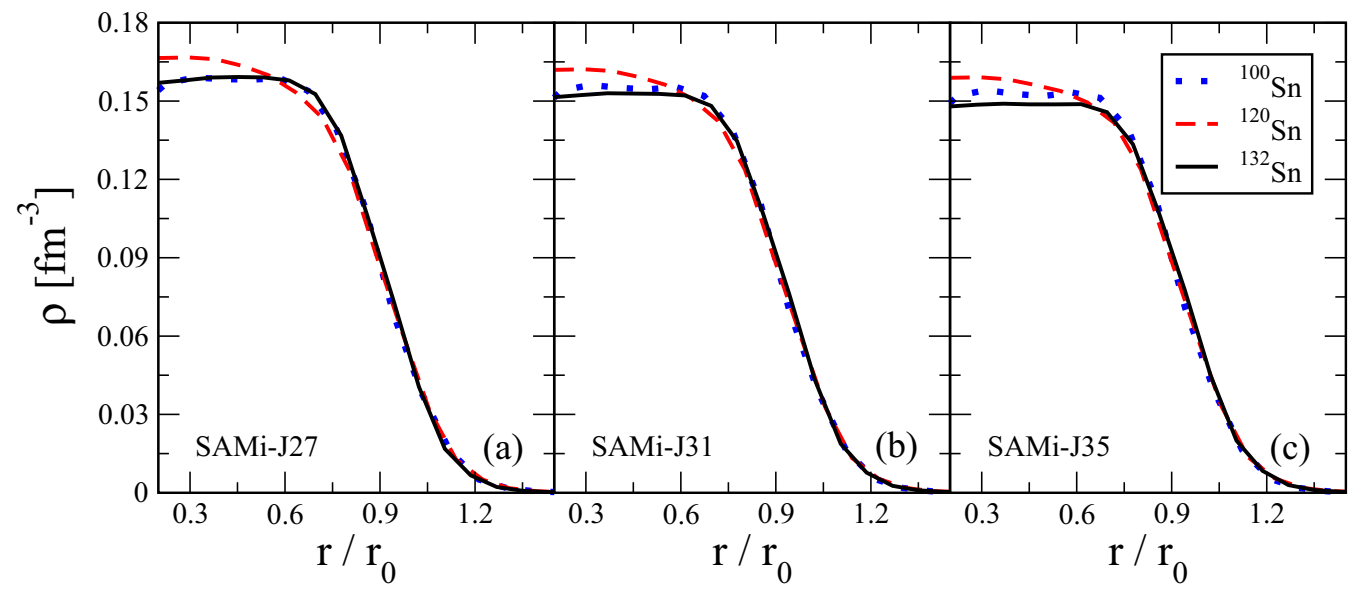

FIG. 12. The isoscalar density profiles of the three spherical nuclei in the Sn isotope chain, for the three parametrizations SAMi-J27 (a), SAMi-J31 (b), and SAMi-J35 (c) considered $\left(r_{0}=1.2 A^{1 / 3}\right)$. 


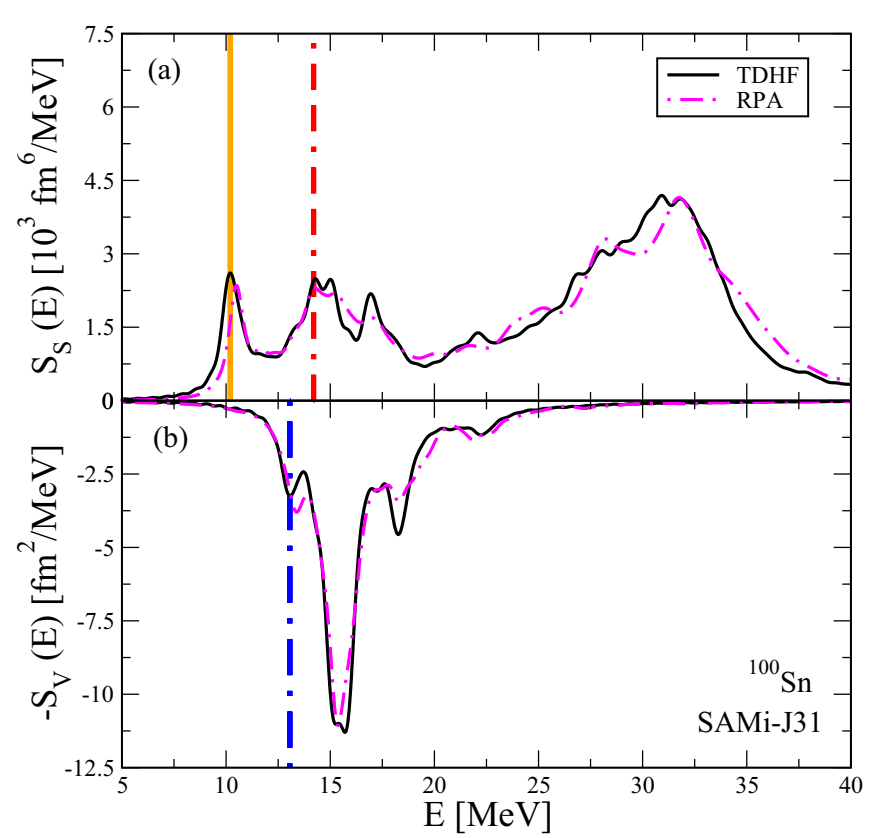

FIG. 13. Similar to Fig. 9, but for ${ }^{100} \mathrm{Sn}$.

renormalized the radius with respect to the standard radius $r_{0}\left(r_{0}=1 \cdot 2 A^{1 / 3}\right)$. From Fig. 12, it is rather evident that, as a consequence of the shell structure, the double magic nuclei exhibit a similar profile, which has a more compact shape and a rather flat behavior in the internal region. This configuration reflects in a sharper radial evolution of the density in the surface region, with respect to the open-shell nucleus ${ }^{120} \mathrm{Sn}$, whose density profile appears more diffuse. At the same time, the isovector density profiles (Fig. 14) clearly show the increasing of the local asymmetry $\rho_{3} / \rho$ in correspondence of the surface, especially for the more neutron-rich system, ${ }^{132} \mathrm{Sn}$, owing to the neutron skin development [74]. This is more evident employing the SAMi-J35 interaction, that has the largest value of the slope L. One can also observe a sligthly proton-rich surface region in the case of ${ }^{100} \mathrm{Sn}$.

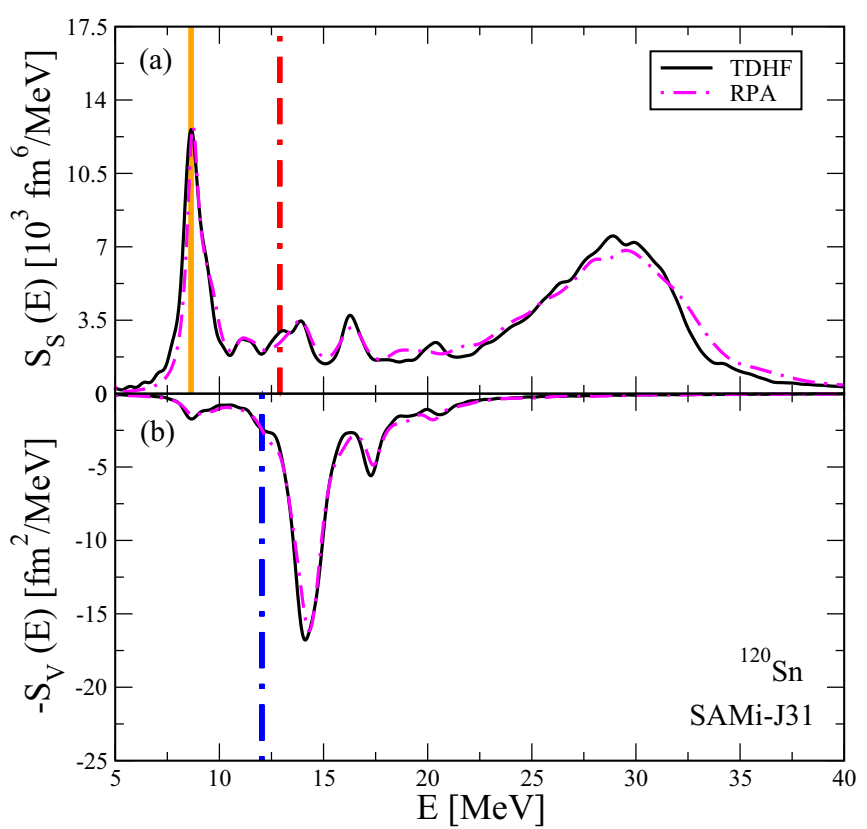

FIG. 15. Similar to Fig. 9, but for ${ }^{120} \mathrm{Sn}$.

Analogously to the analysis presented in Fig. 9 for ${ }^{132} \mathrm{Sn}$, in Figs. 13-15, we show the IS and IV dipole strengths as obtained with both TDHF and RPA models, for ${ }^{100} \mathrm{Sn}$ and ${ }^{120} \mathrm{Sn}$ considering the SAMi-J31 parametrization only. We observe a nice agreement between TDHF and RPA calculations. It clearly emerges that the IS dipole strength of the pygmy mode (indicated by the full orange bar) is strongly enhanced in the case of ${ }^{120} \mathrm{Sn}$, not only with respect to the symmetric system ${ }^{100} \mathrm{Sn}$, but also in comparison to the neutron-rich nucleus ${ }^{132} \mathrm{Sn}$. The relative importance of the pygmy mode in the IS response is in fact enforced at the expense of the strength arising in the energy region just below the IV GDR (peak associated with the dash-dash-dotted red bars in Figs. 9, 13, and 15). As already discussed in Sec. III A, when commenting on the differences observed between Vlasov and

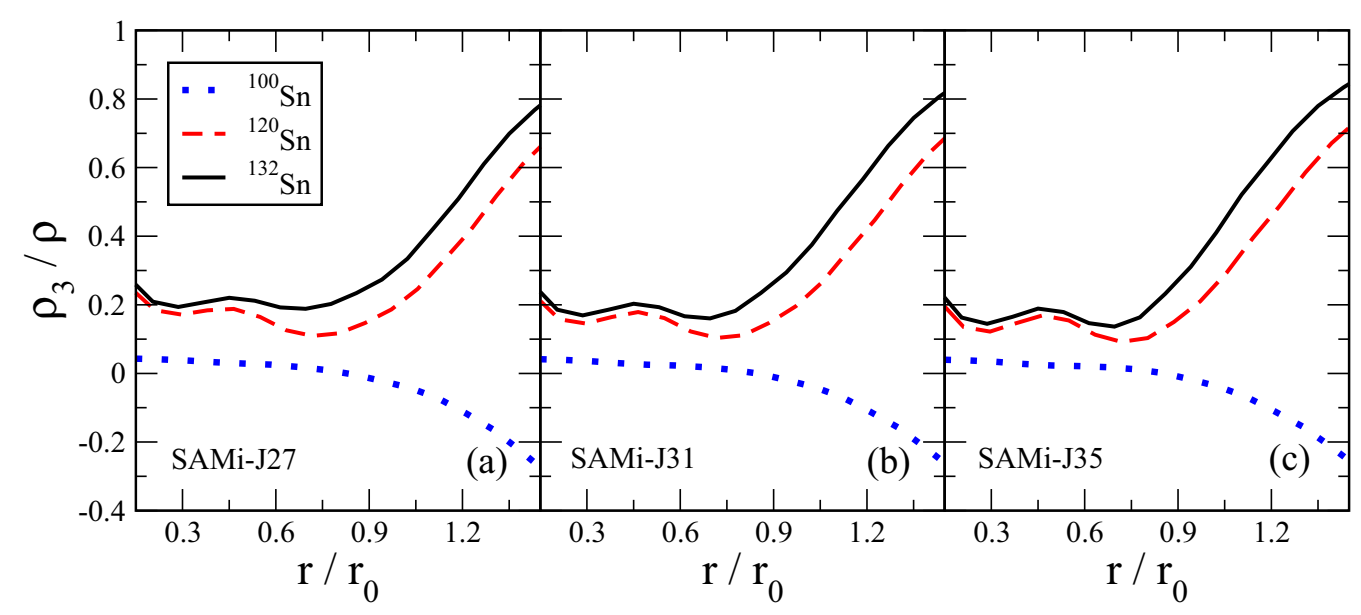

FIG. 14. The local asymmetry profiles of the three spherical nuclei in the Sn isotope chain, for the three parametrizations SAMi-J27 (a), SAMi-J31 (b), and SAMi-J35 (c) considered $\left(r_{0}=1.2 A^{1 / 3}\right)$. 

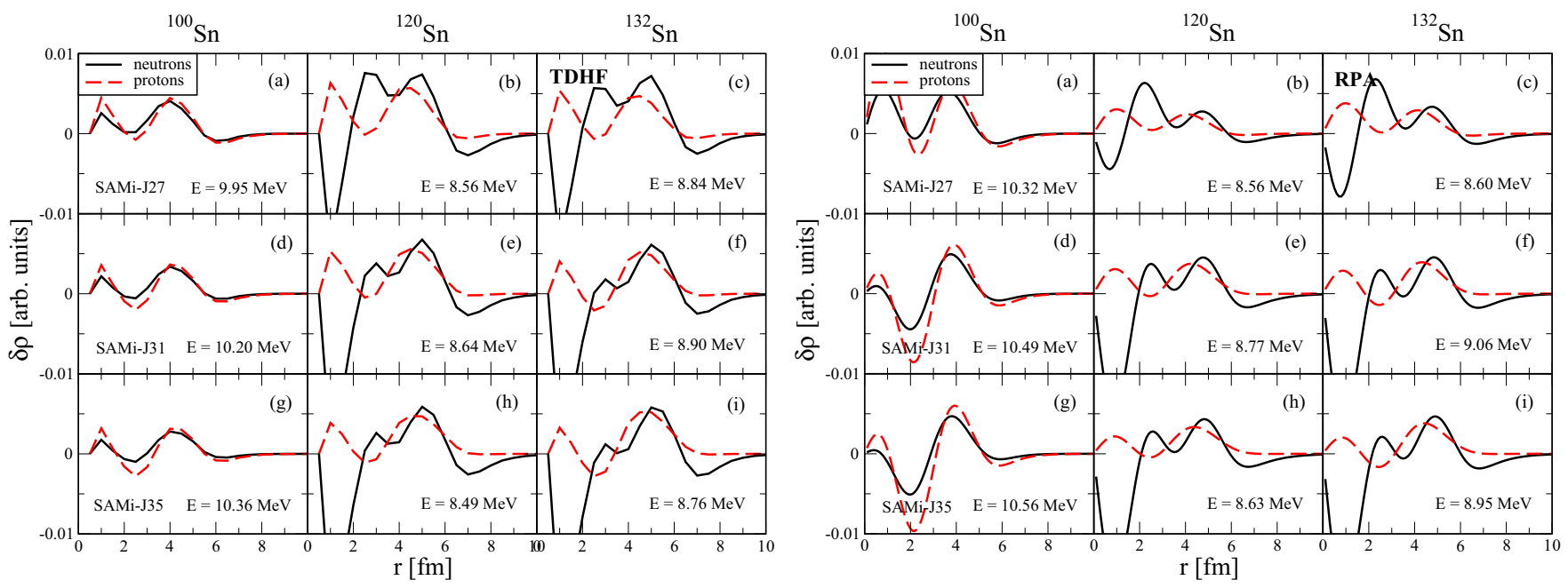

FIG. 16. The transition densities of the PDR (indicated by full orange bars in Figs. 9, 13, and 15), as obtained in TDHF with an initial IS perturbation (left panels) or in RPA (right panels) calculations, for the three nuclei in the Sn isotope chain and for the three SAMi-J parametrizations.

TDHF calculations, this evolution could be connected to the different density profile of the open-shell ${ }^{120} \mathrm{Sn}$, with respect to the closed shell nuclei. Also here, one can notice that a smoother density profile is associated with a larger strength of the isoscalar mode of lowest energy, that has a significant surface component. Owing to the coupling which exists in isospin asymmetric systems, a larger PDS is observed in the IV response. In other words, the PDS does not increase monotonically with $N$, contrarily to the trend exhibited by the neutron-skin in these $\mathrm{Sn}$ isotopes.

Let us concentrate now on the spatial structure of the lowlying energy modes. In the following we will investigate the $\mathrm{Sn}$ isotopes introduced above and we will present the results for the three parametrizations of the effective interaction employed in our study. The left panels in Fig. 16 present the transition densities extracted in TDHF for the lowest energy peak in the pygmy region of the IS dipole response (indicated by full orange bars in Figs. 9, 13, and 15). The result related to the system ${ }^{132} \mathrm{Sn}$ and the SAMi-J31 interaction has already been shown in Fig. 10. However, here our aim is to see the evolution of the PDR structure when varying the isospin asymmetry of the systems, as well as the interaction adopted. First of all, it is interesting to notice once again the isoscalarlike nature of the PDR, especially for the symmetric system, where neutrons and protons oscillate almost exactly in phase. As extensively discussed above, indeed, for this system the isoscalar-isovector mixing, which usually characterizes neutron-rich systems, is strongly reduced. Moreover, in agreement also with our semiclassical results [23], when considering interactions with increasing slope L (from SAMiJ27 to SAMi-J35), one can see that for neutron-rich systems, neutron oscillations become larger, with respect to proton oscillations, especially in the surface region. This can be explained on the basis of Fig. 14, where one observes that the system asymmetry is pushed more towards the surface, corresponding to the development of a thicker neutron skin, when increasing the value of the slope L. The right panels of Fig. 16 show that a good agreement is obtained with the analogous RPA results.

We turn now to examine the peak observed in the IV response (the dash-dotted blue bars in Figs. 9, 13, and 15). As shown by the TDHF calculations of Fig. 17, the transition densities clearly reveal the isovectorlike nature of this mode. Again, as for the PDR, the amplitude of the oscillations at the surface increases as a function of the slope L (see Fig. 7 for the corresponding effect on the strength). Moreover, the IS-IV mixing increases when moving from ${ }^{100} \mathrm{Sn}$ to ${ }^{132} \mathrm{Sn}$. It is interesting to notice that the observation of two lowenergy modes of close energy, the one of lowest energy being mainly isoscalar and the other being mainly isovector, has been reported also in other recent studies [12].

Finally, Fig. 18 shows the transition densities of the second relevant peak appearing in the IS response (that is the peak indicated by dash-dash-dotted red bars in Figs. 9, 13, and 15). As discussed above, this excitation may correspond to the toroidal mode [41]. Indeed, the transition densities deduced by employing an IS perturbation manifest the development of a mode which is clearly isoscalarlike, especially in the case of ${ }^{100} \mathrm{Sn}$ where coupling effects are quenched (see Fig. 18). Also in this case, the IS-IV mixing increases with the symmetry energy slope $L$ of the parametrization considered and with the $N / Z$ of the system. The shape of the transition densities indicates that this mode corresponds to oscillations of the surface against the volume. The small amplitude observed for ${ }^{120} \mathrm{Sn}$, especially in the case of SAMi-J27, is related to the reduced IS strength in the energy region considered (see Fig. 15). It should be noticed that a quite good correspondence with RPA results (not shown here) is obtained also for the modes described in Figs. 17 and 18.

\section{G. Sn isotope chain: Evolution of the PDR strength}

In this section, we aim at assessing the evolution of the PDR strength when varying the $N / Z$ ratio of nuclear systems. 


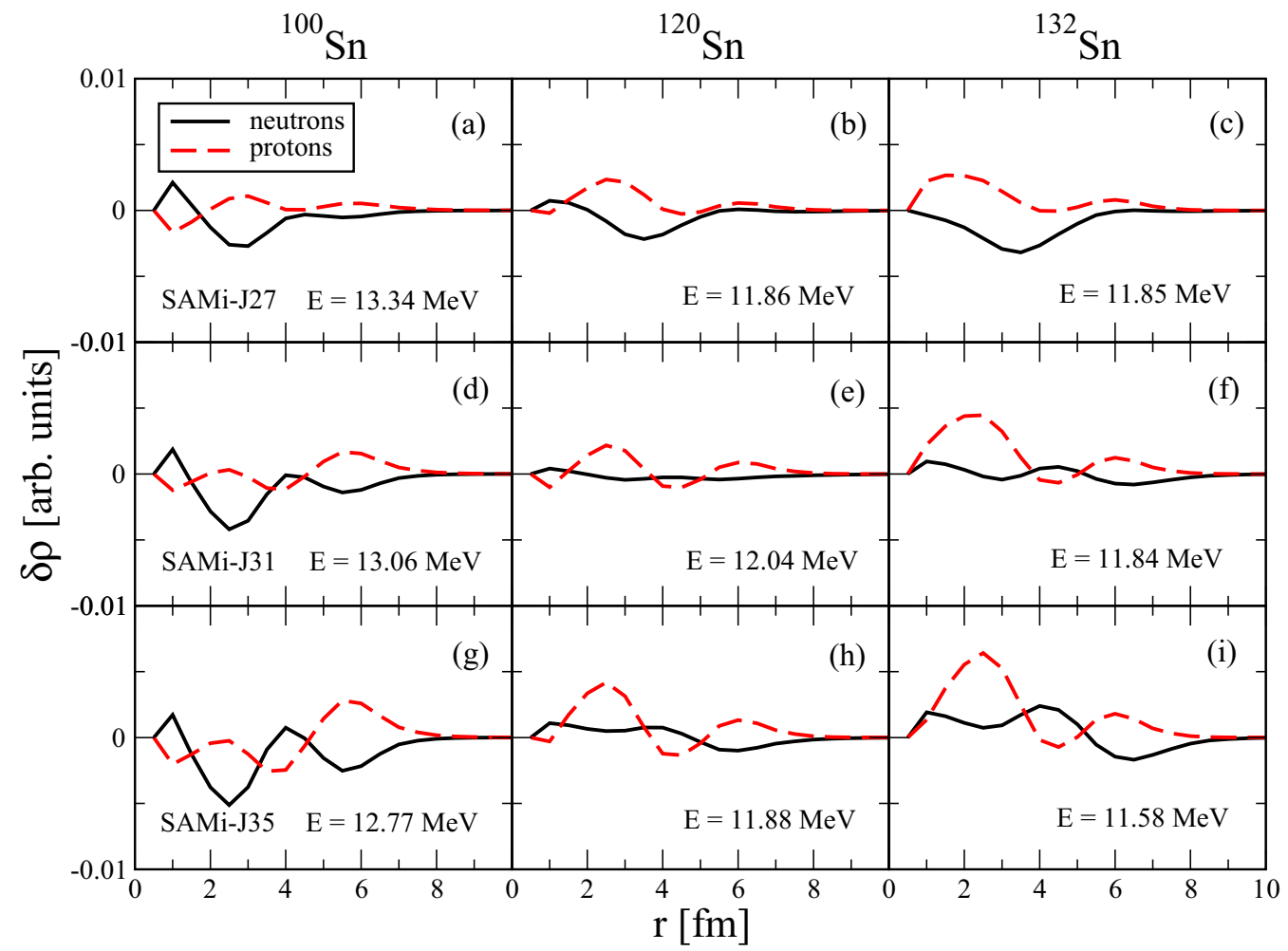

FIG. 17. The transition densities of the second IV peak (indicated by dash-dotted blue bars in Figs. 9, 13, and 15), for the three Sn isotopes. The results are obtained in TDHF and for three SAMi-J parametrizations.

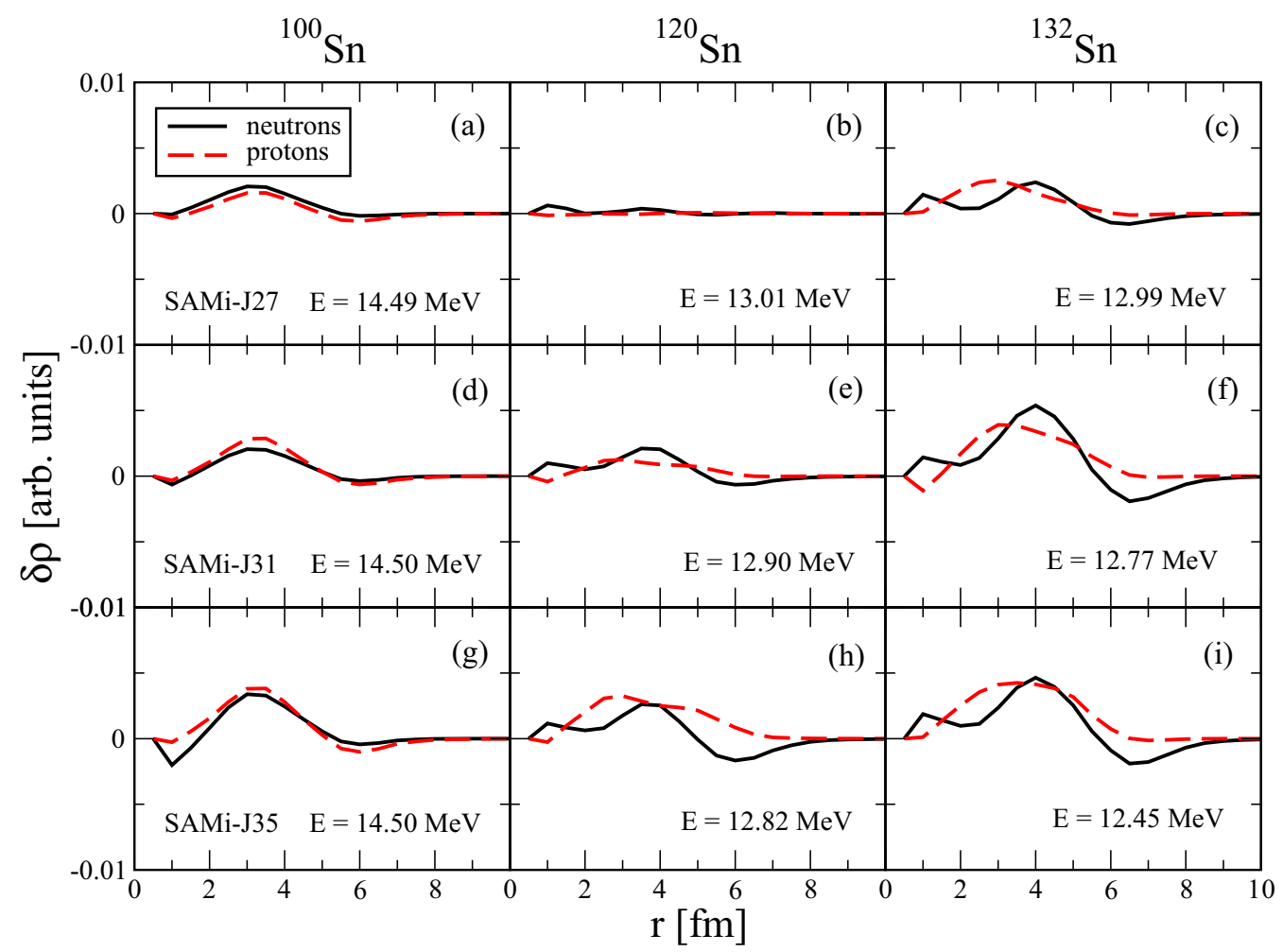

FIG. 18. The transition densities of the second IS peak (dash-dash-dotted red bars in Figs. 9, 13, and 15), for the three Sn isotopes. The results are obtained in TDHF and for three SAMi-J parametrizations. 


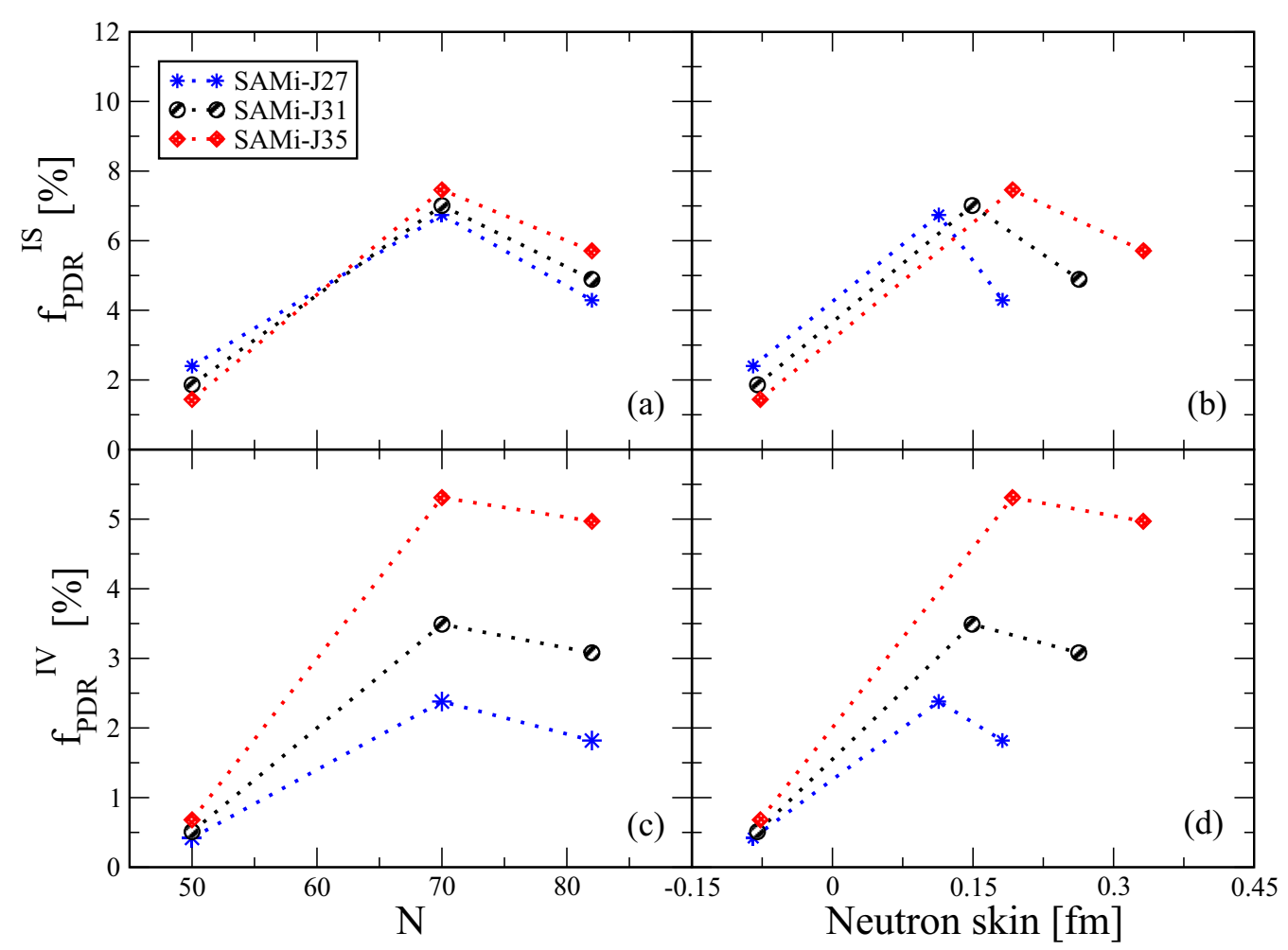

FIG. 19. Fraction of the EWSR exhausted in the PDR region, for the IS (top) and IV (bottom) response, as a function of the neutron number $N$ (left) and the neutron skin (right) of the three Sn isotopes considered.

The analysis developed in recent works [73] looks indeed mostly at the isospin asymmetry dependence of the percentage fraction of the Energy Weigthed Sum Rule (EWSR), $f_{\mathrm{PDR}}$, exhausted in the pygmyw region of the IV dipole response (i.e., by the PDS). Here our goal is instead to establish a connection between the $N / Z$ dependence of the IV dipole response and the concomitant behavior exhibited by the IS dipole strength, in view of the isoscalar-isovector mixing existing in neutron-rich systems and discussed above. As already observed by Ebata et al. [73], despite the increase of the neutron skin thickness, the percentage fraction of the isovector EWSR $f_{\mathrm{PDR}}$ does not grow along the Sn isotope chain, when increasing the neutron number from $N=70$ to $N=82$. This result appears unexpected, considering the relation discussed in several works between the neutron skin thickness and the PDS in the IV dipole response (see Ref. [23] and Refs. therein). Although detailed shell structure effects can be invoked to solve this puzzle, we note that a gateway to this uncommon behavior can be reached on the basis of the isoscalar-isovector mixing discussed so far. The missing fraction in $f_{\mathrm{PDR}}$ could be indeed attributed to the decrease observed in the isoscalar dipole strength, when $\mathrm{Sn}$ isotopes from $N=70$ to $N=82$ are considered. Although the fraction of the EWSR exhausted in the low-energy region of the IV dipole response is expected to increase for nuclei with a larger imbalance in neutron and proton numbers, the result is correlated also to the behavior of the IS response, which in turn reflects the evolution of the isoscalar density profile, when moving from ${ }^{120} \mathrm{Sn}$ to ${ }^{132} \mathrm{Sn}$ (see Fig. 12).
Figure 19 represents the trend of the fraction of the EWSR exhausted in the PDR region (below $10.5 \mathrm{MeV}$ for ${ }^{120} \mathrm{Sn}$ and ${ }^{132} \mathrm{Sn}$ and below $11.3 \mathrm{MeV}$ for ${ }^{100} \mathrm{Sn}$ ) for the IS and IV response, as a function of the neutron number (left) and the neutron skin (right) of the three $\mathrm{Sn}$ isotopes. To better isolate the PDR contribution in the dipole response, a width $\gamma=$ $0.5 \mathrm{MeV}$ has been used in the cut-off function of Eq. (9). One can see that our calculations reproduce the trend discussed, for the IV response (bottom panels), in Ref. [73], with an increase of the EWSR fraction up to $N=70$ and then a decrease, though the neutron skin thickness is larger in nuclei with larger $N$. However, in Fig. 19 (top panels) one can see that the same trend is exhibited also by the IS strength, owing to the prominence of the IS PDR strength in ${ }^{120} \mathrm{Sn}$, as discussed above. It is also interesting to notice that the IS $f_{\mathrm{PDR}}$ does not depend much on the effective interaction considered.

Then, to normalize the effect of the IV mixing to the strength of the mode considered, that is mostly isoscalar, we consider the ratio, $R_{f}$, between the EWSR fractions obtained in the IV and IS response. This quantity is shown in Fig. 20, where a nearly linear increase versus neutron number and neutron skin is now nicely observed. Thus we conclude that, according to the models employed in our study, the evolution of the PDR strength along an isotopic chain is not simply related to the neutron skin thickness. Other ingredients may enter into game as well; indeed a deeper insight into the PDS is got by looking, in parallel, at the corresponding IS strength. Moreover, one can notice that different parametrizations lead 


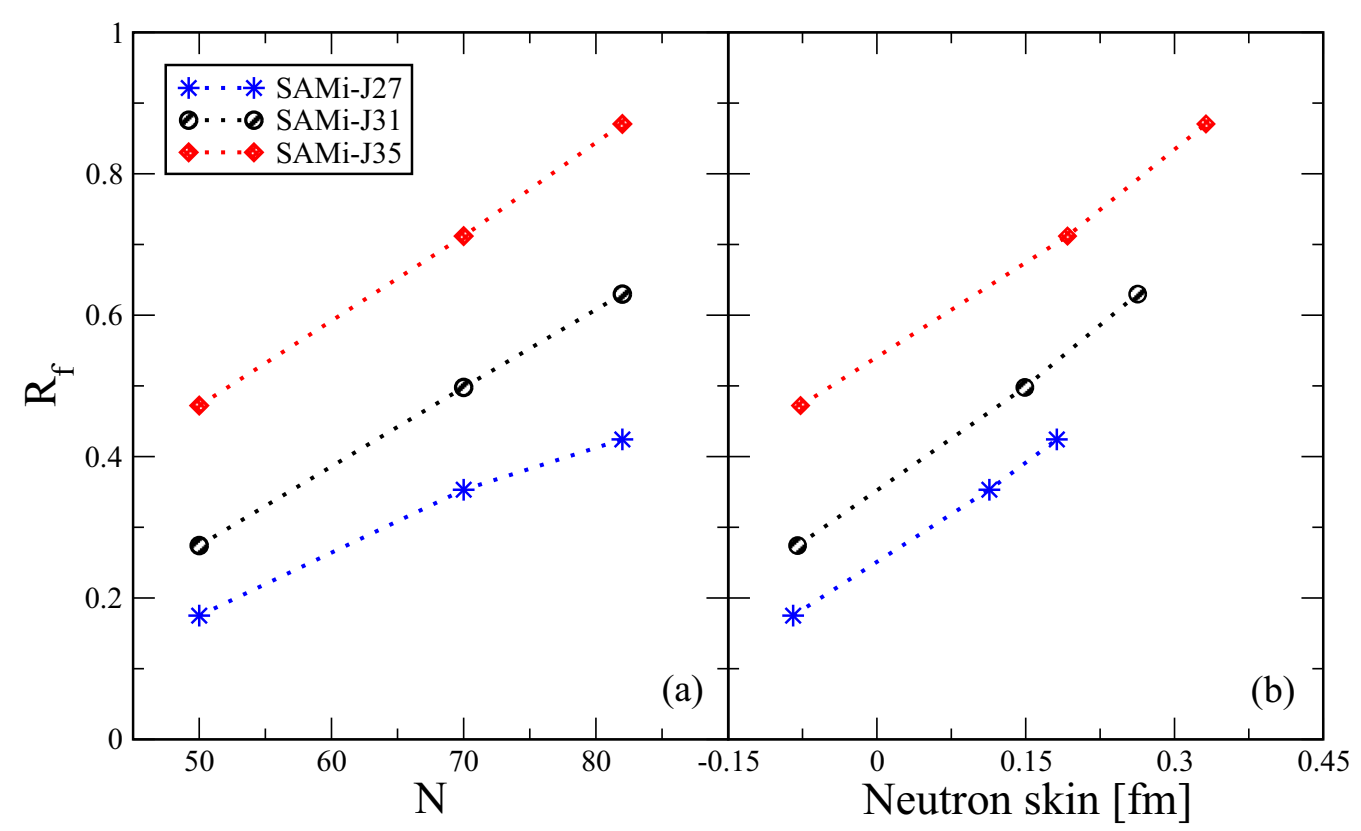

FIG. 20. Ratio between fractions of EWSR exhausted in the PDR region of IV and IS response, as a function of the neutron number $N$ (a) and the neutron skin (b) of the three Sn isotopes considered.

to different results for the $R_{f}$ ratio, even when they predict close values of the neutron skin thickness (see the right panel of Fig. 20). This indicates that, for a given asymmetric system, mixing effects are enhanced for effective interactions with larger symmetry energy slope $L$, as observed in nuclear matter calculations [40].

\section{CONCLUSIONS}

In this article, we have explored the features of the smallamplitude dipole response in selected nuclei within three approaches: TDHF, its zero-amplitude limit (RPA), and its semiclassical limit (Vlasov).

As far as TDHF and RPA calculations are concerned, a detailed comparison of the dipole IS and IV strengths, and of the transition densities of the main excitation modes, is presented here for the first time, showing a good agreement between the results of the two approaches.

The comparison between quantal and semiclassical calculations has evidenced the importance of shell effects and quantal intrinsic gradient terms in shaping isoscalar and isovector density profiles of the ground-state configuration. In particular, HF calculations are generally associated with smoother isoscalar density profiles, with respect to the ones deduced within the TF approximation. Whereas the quantal IV dipole strength is quite well reproduced by Vlasov calculations, significant differences are observed in the low-energy domain of the IS response, concerning the energy and the relative weight of the different peaks. Considering that this region is populated by surface excitation modes, this observation can be reconducted to the different density profiles and the different treatment of surface effects and gradient terms in quantal and semiclassical approaches. Moreover, shell effects can affect significantly the details of the low-lying states, especially as far as the PDS is concerned.

The low-energy region of the dipole response has been investigated in deeper detail. A thorough analysis of the associated transition densities allows one to characterize the different modes in terms of IS-IV mixing and volume/surface components. In particular, we observe that the lowest energy peak, in the PDR region, corresponds to an isoscalarlike surface mode, of larger strength in nuclei with a more diffuse surface. The corresponding IV contribution, i.e., the PDS, originates from mixing effects and increases with the slope L of the symmetry energy. This trend stems from the fact that, as pointed out in several previous investigations (see, for instance, Ref. [69]), a larger L is associated with a neutron enrichment of the surface region; moreover a larger derivative of the symmetry energy also induces stronger IS-IV coupling effects, as indicated by nuclear matter calculations [40]. Then one can argue that, for a considered system, the shape of the low-energy IS response is influenced by the isoscalar density profile, whereas the strength of the corresponding IV counterpart is connected, among other effects, to the surface neutron content, i.e., to the neutron skin thickness. These conclusions hold also in the semiclassical limit. This link to ground-state properties may also help to better understand the impact of relevant terms of the nuclear effective interaction (and nuclear EoS), such as surface gradient terms, compressibility and symmetry energy, on the dipole response features.

Looking at the dipole response of $\mathrm{Sn}$ isotopes, we observe a similar IS strength, in the PDR region, in nuclei with similar density profile (once rescaled by the nuclear radius), such as ${ }^{100} \mathrm{Sn}$ and ${ }^{132} \mathrm{Sn}$, whereas a larger strength appears in the case of ${ }^{120} \mathrm{Sn}$, which exhibits a more diffuse surface. The 
corresponding IV projection follows a similar behavior, being larger in the ${ }^{120} \mathrm{Sn}$ case, in spite of the thicker neutron-skin of ${ }^{132} \mathrm{Sn}$. An increasing trend with the neutron skin thickness can be recovered if one considers the ratio between the IV and IS EWSR fractions exhausted by the PDR region.

\section{ACKNOWLEDGMENT}

Funding from the European Union's Horizon 2020 research and innovation program under Grant No. 654002 is acknowledged.
[1] A. Bohr and B. Mottelson, Nuclear Structure 1 (W. A. Benjamin, San Francisco, 1969).

[2] D. J. Rowe, Nuclear Collective Motion: Models and Theory (World Scientific, Singapore, 2010).

[3] D. A. Butts and D. S. Rokhsar, Nature 397, 327 (1999).

[4] K. W. Madison, F. Chevy, W. Wohlleben, and J. Dalibard, Phys. Rev. Lett. 84, 806 (2000).

[5] O. M. Marago, S. A. Hopkins, J. Arlt, E. Hodby, G. Hechenblaikner, and C. J. Foot, Phys. Rev. Lett. 84, 2056 (2000).

[6] T. H. Oosterkamp, J. W. Janssen, L. P. Kouwenhoven, D. G. Austing, T. Honda, and S. Tarucha, Phys. Rev. Lett. 82, 2931 (1999).

[7] M. Toreblad, M. Borgh, M. Koskinen, M. Manninen, and S. M. Reimann, Phys. Rev. Lett. 93, 090407 (2004).

[8] G. C. Baldwin, G. S. Klaiber, Phys. Rev. 71, 3 (1947).

[9] N. Paar, T. Nikšić, D. Vretenar, and P. Ring, Phys. Lett. B 606, 288 (2005).

[10] D. Savran et al., Phys. Rev. C 84, 024326 (2011).

[11] F. C. L. Crespi et al., Phys. Rev. Lett. 113, 012501 (2014).

[12] A. Bracco, F. C. L. Crespi, and E. G. Lanza, Eur. Phys. J. A 51, 99 (2015).

[13] N. Paar, D. Vretenar, E. Khan, and G. Colò, Rep. Prog. Phys. 70, 691 (2007).

[14] D. Savran, T. Aumann, and A. Zilges, Prog. Part. Nucl. Phys. 70, 210 (2013).

[15] P.-G. Reinhard and W. Nazarewicz, Phys. Rev. C 87, 014324 (2013).

[16] L. Pellegri et al., Phys. Lett. B 738, 519 (2014).

[17] F. C. L. Crespi et al., Phys. Rev. C 91, 024323 (2015).

[18] I. Poltoratska et al., Phys. Rev. C 85, 041304(R) (2012).

[19] A. M. Krumbholz et al., Phys. Lett. B 744, 7 (2015).

[20] D. Savran, M. Babilon, A. M. van den Berg, M. N. Harakeh, J. Hasper, A. Matic, H. J. Wortche, and A. Zilges, Phys. Rev. Lett. 97, 172502 (2006).

[21] J. Endres, D. Savran, A. M. vandenBerg, P. Dendooven, M. Fritzsche, M. N. Harakeh, J. Hasper, H. J. Wortche, and A. Zilges, Phys. Rev. C 80, 034302 (2009).

[22] P. Papakonstantinou, H. Hergert, V. Yu. Ponomarev, and R. Roth, Phys. Rev. C 89, 034306 (2014).

[23] H. Zheng, S. Burrello, M. Colonna, and V. Baran, Phys. Rev. C 94, 014313 (2016).

[24] D. Lacroix, S. Ayik, and P. Chomaz, Prog. Part. Nucl. Phys. 52, 497 (2004).

[25] C. Simenel, B. Avez, and D. Lacroix, Quantum Many-Body Dynamics: Applications to Nuclear Reactions (VDM Verlag, Sarrebruck, 2010).

[26] T. Nakatsukasa, K. Matsuyanagi, M. Matsuo, and K. Yabana, Rev. Mod. Phys. 88, 045004 (2016).

[27] P. Ring and P. Schuck, The Nuclear Many-Body Problem (Springer-Verlag, Berlin, 1980).

[28] D. M. Brink, A. Dellafiore, and M. Di Toro, Nucl. Phys. A 456, 205 (1986)
[29] G. F. Burgio and M. Di Toro, Nucl. Phys. A 476, 189 (1988).

[30] V. I. Abrosimov, A. Dellafiore, and F. Matera, Nucl. Phys. A 697, 748 (2002).

[31] P. Ring, Z. Y. Ma, N. Van Giai, D. Vretenar, A. Wandelt, and L. G. Cao, Nuc. Phys. A 694, 249 (2001).

[32] D. Vretenar, H. Berghammer, and P. Ring, Nuc. Phys. A 581, 679 (1995)

[33] D. Vretenar, A. V. Afanasjev, G. A. Lalazissis, and P. Ring, Phys. Rep. 409, 101 (2005).

[34] V. Baran, M. Colonna, M. Di Toro, A. Croitoru, and D. Dumitru, Phys. Rev. C 88, 044610 (2013); V. Baran, D. I. Palade, M. Colonna, M. DiToro, A. Croitoru, and A. I. Nicolin, ibid. 91, 054303 (2015).

[35] X. Roca-Maza, M. Brenna, B. K. Agrawal, P. F. Bortignon, G. Colo, L. G. Cao, N. Paar, and D. Vretenar, Phys. Rev. C 87, 034301 (2013).

[36] X. Roca-Maza and N. Paar, Prog. Part. Nucl. Phys. 101, 96 (2018).

[37] E. Yüksel, G. Colò, E. Khan, and Y. F. Niu, Phys. Rev. C 97, 064308 (2018).

[38] C. Simenel and S. Umar, Prog. Part. Nucl. Phys. 103, 19 (2018)

[39] G. Colò, L. Cao, N. Van Giai, and L. Capelli, Comput. Phys. Commun. 184, 142 (2013).

[40] V. Baran, M. Colonna, V. Greco, and M. Di Toro, Phys. Rep. 410, 335 (2005).

[41] M. Urban, Phys. Rev. C 85, 034322 (2012).

[42] V. Baran, B. Frecus, M. Colonna, and M. Di Toro, Phys. Rev. C 85, 051601(R) (2012).

[43] Ad. R. Raduta et al., Eur. Phys. J. A 50, 24 (2014).

[44] H. Zheng, S. Burrello, M. Colonna, D. Lacroix, and G. Scamps, Phys. Rev. C 98, 024622 (2018)

[45] X. Roca-Maza, G. Colò, and H. Sagawa, Phys. Rev. C 86, 031306(R) (2012).

[46] L. G. Cao, G. Colò, and H. Sagawa, Phys. Rev. C 81, 044302 (2010); 72, 067303 (2005); Phys. Lett. B 455, 25 (1999).

[47] R. B. Wiringa, V. Fiks, and A. Fabrocini, Phys. Rev. C 38, 1010 (1988).

[48] X. Roca-Maza, N. Paar, and G. Colò, J. Part. Nucl. Phys. G 42, 034033 (2015).

[49] P. Bonche, H. Flocard, and P. H. Heenen, Comput. Phys. Commun. 171, 49 (2005).

[50] P. Möller, A. J. Sierk, T. Ichikawa, and H. Sagawa, At. Data Nucl. Data Tables 109-110, 1 (2016).

[51] G. Scamps and D. Lacroix, Phys. Rev. C 88, 044310 (2013).

[52] G. Scamps, Effet de l'appariement sur la dynamique nucléaire., Diss. Université de Caen (2014).

[53] X. Roca-Maza, X. Viñas, M. Centelles, B. K. Agrawal, G. Colò, N. Paar, J. Piekarewicz, and D. Vretenar, Phys. Rev. C 92, 064304 (2015).

[54] C. Y. Wong, Phys. Rev. C 25, 1460 (1982).

[55] A. Guarnera, M. Colonna, and Ph. Chomaz, Phys. Lett. B 373, 297 (1996). 
[56] F. Calvayrac, P. G. Reinhard, and E. Suraud, Ann. Phys. 225, 125 (1997).

[57] V. I. Abrosimov and O. I. Davydovs'ka, Ukr. J. Phys. 54, 1068 (2009); V. Baran et al., Rom. J. Phys. 57, 36 (2012).

[58] K.-H. Kim, T. Otsuka, and P. Bonche, J. Phys. G 23, 1267 (1997).

[59] D. Lacroix, arXiv:nucl-th/0202063.

[60] G. Scamps and D. Lacroix, Phys. Rev. C 89, 034314 (2014).

[61] P.-G. Reinhard, P. D. Stevenson, D. Almehed, J. A. Maruhn, and M. R. Strayer, Phys. Rev. E 73, 036709 (2006).

[62] C. W. De Jager et al., At. Data Nucl. Data Tables 36, 495 (1987);

G. Audi and A. H. Wapstra, Nucl. Phys. A 595, 409 (1995); I. Angeli and K. P. Marinova, At. Data Nucl. Data Tables 99, 69 (2013).

[63] B. K. Jennings, Phys. Lett. B 74, 13 (1978).

[64] V. B. Soubbotin and X. Viñas, Nuc. Phys. A 665, 291 (2000).
[65] D. Vretenar, Y. F. Niu, N. Paar, and J. Meng, Phys. Rev. C 85, 044317 (2012).

[66] X. Roca-Maza, G. Pozzi, M. Brenna, K. Mizuyama, and G. Colò, Phys. Rev. C 85, 024601 (2012).

[67] A. Repko, P.-G. Reinhard, V. O. Nesterenko, and J. Kvasil, Phys. Rev. C 87, 024305 (2013).

[68] L. Trippa, G. Colò, and E. Vigezzi, Phys. Rev. C 77, 061304(R) (2008).

[69] A. Carbone, G. Colo, A. Bracco, L. G. Cao, P. F. Bortignon, F. Camera, and O. Wieland, Phys. Rev. C 81, 041301(R) (2010).

[70] J. Piekarewicz, Phys. Rev. C 83, 034319 (2011).

[71] M. Goldhaber and E. Teller, Phys. Rev. 74, 1046 (1948).

[72] H. Steinwedel and J. H. D. Jensen, Phys. Rev. 79, 1019 (1950).

[73] S. Ebata, T. Nakatsukasa, and T. Inakura, Phys. Rev. C 90, 024303 (2014).

[74] A. Trzcinska, J. Jastrzebski, P. Lubinski, F. J. Hartmann, R. Schmidt, T. von Egidy, and B. Klos, Phys. Rev. Lett. 87, 082501 (2001). 